This item was submitted to Loughborough's Research Repository by the author.

Items in Figshare are protected by copyright, with all rights reserved, unless otherwise indicated.

\title{
Positivity of divisors on blown-up projective spaces, II
}

PLEASE CITE THE PUBLISHED VERSION

https://doi.org/10.1016/j.jalgebra.2019.02.041

PUBLISHER

(c) Elsevier

VERSION

AM (Accepted Manuscript)

PUBLISHER STATEMENT

This paper was accepted for publication in the journal Journal of Algebra and the definitive published version is available at https://doi.org/10.1016/j.jalgebra.2019.02.041.

\section{LICENCE}

CC BY-NC-ND 4.0

\section{REPOSITORY RECORD}

Postinghel, Elisa, and Olivia Dumitrescu. 2019. "Positivity of Divisors on Blown-up Projective Spaces, II". figshare. https://hdl.handle.net/2134/37403. 


\title{
POSITIVITY OF DIVISORS ON BLOWN-UP PROJECTIVE SPACES, II
}

\author{
OLIVIA DUMITRESCU AND ELISA POSTINGHEL
}

\begin{abstract}
We construct log resolutions of pairs on the blow-up of the projective space in an arbitrary number of general points and we discuss the semi-ampleness of the strict transforms. As an application we give an explicit proof that the abundance conjecture holds for an infinite family of such pairs.

For $n+2$ points, these strict transforms are F-nef divisors on the moduli space $\overline{\mathcal{M}}_{0, n+3}$ in a Kapranov's model: we show that all of them are nef.
\end{abstract}

\section{Contents}

Introduction

Part 1. Linearly obstructed divisors 3

1. Notation, known results and conjectures 3

1.1. The cohomology of divisors on $X_{s,(r)}$

2. Globally generated divisors on $X_{s,(r)} \quad 6$

2.1. Vanishing cohomology of strict transforms 11

2.2. Log resolutions for divisors on blown-up projective spaces in points 13

3. Log abundance for linearly obstructed divisors on $X_{s,(0)} \quad 13$

3.1. Proof of Theorem 3.3, only linearly obstructed case 15

4. On the F-conjecture 16

4.1. Preliminaries and notation $\quad 17$

4.2. The F-conjecture holds for strict transforms on $\overline{\mathcal{M}}_{0, n} \quad 19$

Part 2. Divisors obstructed by rational curves and their secants 20

5. Notation and preliminary results 20

5.1. Strong base locus lemma for divisors on $X_{s+3,(0)} \quad 21$

5.2. The blown-up space $X_{n+3,(n-2)}^{\sigma} \quad 22$

5.3. Conjectures on vanishing cohomology for divisors on $X_{n+3,(n-2)}^{\sigma} \quad 23$

6. Explicit proof of log abundance for effective divisors on $X_{n+3,(0)} \quad 24$

6.1. Constructing a log resolution of $(X, \epsilon D) \quad 25$

6.2. Proof of Theorem 6.10, case $n$ even 28

6.3. Proof of Theorem 6.10, case $n$ odd 30

References $\quad 32$

2010 Mathematics Subject Classification. Primary: 14C20 Secondary: 14C17, 14J70.

Key words and phrases. globally generated divisors, abundance conjecture, F-conjecture.

The first author is supported by the Simons Foundation travel grant 524545 . 


\section{INTRODUCTION}

This paper studies positivity questions for divisors on blow-ups of projective spaces of arbitrary dimension in points in general position and along the linear cycles these points span.

Let $X_{s,(0)}$ denote the blow-up of $\mathbb{P}^{n}$ at $s$ points in general position. An interesting problem is the dimensionality problem that consists in the computation of dimension of the space of global sections of the sheaves associated to effective divisors on $X_{s,(0)}$. This is related to the polynomial interpolation problem for homogeneous polynomials of fixed degree and multiplicity at a collection of $s$ general points. This problem is longstanding and is related to the Nagata's conjecture and the Segre-Harbourne-Gimigliano-Hirschowitz conjecture for $n=2$, see [11] for an account, and to Fröberg-Iarrobino conjectures for ideals generated by forms. In [5] the authors solved this problem for divisors on $X_{s,(0)}$ for $s \leq n+2$ and in [6] they gave a conjectural formula for the dimension of linear systems on $X_{n+3,(0)}$ that takes into account the contributions given by the rational normal curve and the joins of its secants with linear subspaces. In [13] further positivity properties such as basepoint freeness and, more generally, $l$-very ampleness of linear series on $X_{s,(0)}$, for arbitrary $s$, are studied: these have "large" degree with respect to the multiplicities of the points.

This manuscript analyses all other linear series, i.e. those with low degree whose general member has singularities. In particular we construct resolutions of these singularities and we prove that proper transform of divisors are basepoint free.

Let $X_{s,(n-2)}$ denote the subsequent blow-up of $X_{s,(0)}$ along all the linear subspaces of $\mathbb{P}^{n}$ spanned by the $s$ points in increasing dimension. Of particular interest are the cases $s=n+1$ and $s=n+2$ that correspond to the compactifications of the Losev-Manin moduli space (see [27]) and of the moduli space of stable rational marked curves $\overline{\mathcal{M}}_{0, n+3}$ (see [22]) respectively. In this article we pose a series of questions about vanishing cohomology of strict transforms on $X_{s,(n-2)}$ of divisors on $X_{s,(0)}$ of which a positive answer would imply a solution to the dimensionality problem, see Subsection 5.2.

We recall that a divisor $D$ on $X_{s,(0)}$ is said to be only linearly obstructed if the dimension of the corresponding linear system equals its linear expected dimension introduced in [5]. In [5, 12] it was shown that all effective as well as certain non effective divisors $D$, that are only linearly obstructed, can be birationally modified, by blowing-up their linear base locus and subtracting the linear divisorial components; this produced divisors $\tilde{D}$, the strict transforms of $D$, whose higher cohomology groups vanish. An application of these vanishing theorems that will be developed in this paper is the description of globally generated and semi-ample divisors (Theorem 2.1). Furthermore we extend this result to a class of divisors on $X_{n+3,(0)}$ that can have non-linear base locus, by taking the blow-up along the joins between linear cycles and secant varieties to the unique rational normal curve of degree $n$ through the $s$ points (Theorem 6.10).

The varieties $X_{n+3,(0)}$ were very much studied recently as a source of interesting and explicit examples of Fano type manifolds in higher dimension. If $s \leq n+3$, $X_{s,(0)}$ is $\log$ Fano, hence in particular a Mori dream space (see for example [2, 9]). The space $X_{n+3,(0)}$ is of particular interest because it is the moduli space of parabolic vector bundles of rank 2 over $\mathbb{P}^{1}$ (see [3], [30, 28]). The Cox ring $X_{n+3,(0)}$, end therefore extremal rays of the effective cone of divisors, was given in [9]. Equations 
for the facets of the effective cone and the movable cone of divisors were computed by the two authors of this manuscript together with Brambilla in [6]. These were found based on a base locus lemma for joins between linear subspaces spanned by the points and the secant varieties of the unique rational normal curve of degree $n$ determined by the collection of $n+3$ points (Lemma 5.2). For $n$ even, $X_{n+3,(0)}$ is isomorphic in codimension one to the $n$-dimensional Fano variety of $\left(\frac{n}{2}-1\right)$-planes in a smooth complete intersection of two quadrics in $\mathbb{P}^{n+2}$, studied recently in [1]. Araujo and Massarenti [2] recently gave an explicit log Fano structure to the blowup of $\mathbb{P}^{n}$ in up to $n+3$ points in general position; they do so by studying the blow-up $X_{n+3,(n-2)}^{\sigma}$ of $\mathbb{P}^{n}$ along the joins mentioned above.

In this article, we prove a number of results in birational geometry.

The log abundance conjecture is one of the main open questions in the birational classification of higher dimensional algebraic varieties. It predicts that for every pair given by a projective variety $X$ and an effective divisor $\Delta$ on $X$ with $\log$ canonical singularities, if the adjoint bundle $K_{X}+\Delta$ is nef, then it is semi-ample (cf. Conjecture 3.2). It is known to hold for surfaces and threefolds (see [23]) and for fourfolds with positive Kodaira dimension (see [14]). Moreover, it was recently proved to hold for rationally connected varieties (see [17] and [19]), otherwise very little is known in higher dimension. A precise classification of divisors on the blowup of $\mathbb{P}^{n}$ in collections of points in general position, for which abundance holds, is not easily deduced from the work contained in these references. In this paper we construct explicitly infinite families of $\log$ pairs $\left(X_{s,(0)}, D\right)$, with $D$ effective, for which abundance holds, see Theorems 3.3 and 6.1.

As explained in [5, Subsection 6.3], the F-conjecture predicting the nef cone of $\overline{\mathcal{M}}_{0, n}$ was the original motivation for the study of the vanishing theorems. A divisor on $\overline{\mathcal{M}}_{0, n}$ intersecting non-negatively the one dimensional strata of $\overline{\mathcal{M}}_{0, n}$ is called $F$-nef. The F-conjecture proposed by Fulton, states that a divisor on $\overline{\mathcal{M}}_{0, n}$ is nef if and only if is F-nef.

As noted above, the iterated blow-up $X_{n+2,(n-2)}$ of the projective space is identified with the moduli space $\overline{\mathcal{M}}_{0, n+3}$. Using vanishing theorems we prove that the conjecture holds for strict transforms of linear systems in $\mathbb{P}^{n}$ interpolating multiple points in general position, under the blow-up of their linear base locus, Theorem 4.8 .

This paper is organized as follows.

In part 1 we study properties of divisors on $X_{s,(0)}$, that have only linear base locus, and of their strict transforms in further blow-ups $X_{s,(n-2)}$. In Part 2 we study all effective divisors on $X_{n+3,(0)}$, that contain linear and non-liner obstructions, and we study properties of their strict transform in $X_{n+3,(n-2)}^{\sigma}$, the iterated blow-up of all cycles of the base locus.

In Section 1 we introduce the general construction, notation and some preliminary facts.

Section 2 studies semi-ampleness of divisors on $X_{s,(n-2)}$, the main result is Theorem 2.1. Subsection 2.1 contains a complete description of the base locus of only linearly obstructed linear systems in $\mathbb{P}^{n}$ interpolating multiple points, Theorem 2.6.

In Sections 3 we prove that the (log) abundance conjecture holds for log pairs on $X_{s,(0)}$ that have only linear base locus, by taking log resolutions $X_{s,(n-2)}$, Theorem 3.3 
In Section 4, as an application of the results contained in Section 2, we establish the F-conjecture for a particular class of divisors, Theorem 4.8.

In Section 5 we construct $\log$ resolutions $X_{n+3,(n-2)}^{\sigma}$ of effective divisors on $X_{n+3,(0)}$. In Subsection 5.2 we pose a number of questions about the cohomology of the strict transform of these divisors.

In Section 6 we prove that log abundance conjecture holds for pairs on $X_{n+3,(0)}$ that are not only linearly obstructed, by taking $\log$ resolutions $X_{n+3,(n-2)}^{\sigma}$.

\section{Part 1. Linearly obstructed divisors}

\section{Notation, KNOWN RESUlts AND CONJECTURES}

Let $K$ be an algebraically closed field of characteristic zero. Let $\mathcal{S}=\left\{p_{1}, \ldots, p_{s}\right\}$ be a collection of $s$ distinct points in $\mathbb{P}_{K}^{n}$ and let $S$ be the set of indices parametrizing $\mathcal{S}$, with $|S|=s$.

Notation 1.1. (Interpolation problems) Let

$$
\mathcal{L}:=\mathcal{L}_{n, d}\left(m_{1}, \ldots, m_{s}\right)
$$

denote the linear system of degree- $d$ hypersurfaces of $\mathbb{P}^{n}$ with multiplicity at least $m_{i}$ at $p_{i}$, for $i=1, \ldots, s$.

Notation 1.2. We denote by $X_{s,(0)}$ the blow-up of $\mathbb{P}^{n}$ in the points $\mathcal{S}$ and by $E_{i}$ the exceptional divisor of $p_{i}$, for all $i$. The index (0) indicates that the space $\mathbb{P}^{n}$ is blown-up in 0-dimensional cycles. The Picard group of $X_{s,(0)}$ is spanned by the class of a general hyperplane, $H$, and the classes of the exceptional divisors $E_{i}$, $i=1, \ldots, s$.

Notation 1.3. Fix positive integers $d, m_{1}, \ldots, m_{s}$ and define the following divisor on $X_{s,(0)}$ :

$$
d H-\sum_{i=1}^{s} m_{i} E_{i} \in \operatorname{Pic}\left(X_{s,(0)}\right) .
$$

Let $D$ be a general divisor in $\left|d H-\sum_{i=1}^{s} m_{i} E_{i}\right|$.

For every $I \subset \mathcal{S}$, we set $|I|=: \rho+1$ and we define the integer

$$
k_{I}=k_{I}(D):=\max \left\{0, m_{i_{1}}+\cdots+m_{i_{\rho+1}}-\rho d\right\} .
$$

that computes the multiplicity of containment of $L_{I}$ in $\operatorname{Bs}(|D|)$, see $[12$, Proposition $4.2]$.

Notation 1.4. For $-1 \leq r \leq \min \{s, n\}-1$, we denote by $X_{s,(r)}$ the iterated blowup of $\mathbb{P}^{n}$ along the strict transform of the linear cycles $L_{I}$ of dimension less than or equal to $r$ spanned by sets of points $I \subset \mathcal{S},|I| \leq r+1$, with $k_{I}>0$, ordered by increasing dimension. We further denote by $E_{I}$ the (strict transform of) the exceptional divisor in $X_{s,(r)}$ of the linear space $L_{I}$, for every such an $I$.

When $r=0, X_{s,(0)}$ is the blow-up introduced in Notation 1.2. If $r=-1$ then we adopt the convention $X_{s,(-1)}:=\mathbb{P}^{n}$.

We point out that if $r \geq 1$ the space $X_{s,(r)}$ depends on the divisor $D$, precisely on the integers $k_{I}$, but for the sake of simplicity we decided to omit this dependency from the notation. If $D$ is an effective divisor on $X_{s,(0)}$, the birational morphism

$$
X_{s,(r)} \rightarrow X_{s,(0)}
$$


obtained as composition of blow-ups, resolves the linear base locus of $D$ (see [5, Section 4] and [12, Section 2]).

Remark 1.5. Abusing notation, we will denote by $L_{I}$ the linear subspace of $X_{s,(-1)}$ spanned by the set of points parametrized by $I$ as well as its strict transform in the blown-up spaces $X_{s,(r)}$, for every $r \geq 0$.

(1) For $|I| \geq r+2, L_{I}$ on $X_{s,(r)}$ represents a blown-up projective space of dimension $|I|-1$ along linear cycles of dimension at most $r$.

(2) If $|I| \leq r+1$, the strict transform of $L_{I}$ will be the exceptional divisor $E_{I}$ that is a product of blown-up projective spaces. The full description of this space and its intersection theory is explicitly given in [12, Section 2].

Notation 1.6. Denote by $D_{(r)}$ the strict transform of the divisor $D$ on $X_{s,(r)}$. One has

$$
D_{(r)}:=d H-\sum_{\substack{I \subset\{1, \ldots, s\}: \\ 0 \leq|I| \leq r+1}} k_{I} E_{I},
$$

where the integers $k_{I}$ are defined in (1.3).

Remark 1.7. If $r=n-1$, then $D_{(n-1)}$ is the strict transform of $D_{(n-2)}$ via $X_{s,(n-2)} \rightarrow X_{s,(n-2)}$, that is the divisor on $X_{s,(n-2)}$ obtained from $D_{(n-2)}$ by subtracting $k_{I(n-1)}$ times the strict transform of the fixed hyperplane spanned by the points parametrized by $I(n-1)$, for any $I(n-1) \subset\{1, \ldots, s\}$, namely those that are fixed components of $D_{(n-2)}$.

Notation 1.8. Set $\bar{r}$ to be the maximal dimension of the linear cycles of the base locus of $D$. We will also use $\tilde{D}:=D_{(\bar{r})}$ to denote the strict transform of $D$ under the blow-up of all its linear base locus (including subtraction of hyperplanes for $\bar{r}=n-1$, as in Remark 1.7).

1.1. The cohomology of divisors on $X_{s,(r)}$. In this section we discuss the cohomology spaces $H^{i}\left(X_{s,(r)}, \mathcal{O}_{X_{s,(r)}}\left(D_{(r)}\right)\right)$. To simplify notation we will abbreviate $\operatorname{dim} H^{i}\left(X_{s,(r)}, \mathcal{O}_{X_{s,(r)}}\left(D_{(r)}\right)\right)$ by $h^{i}\left(D_{(r)}\right)$.

Let $s(d)$ denote the number of points with multiplicity $d$ of $D$, namely the number of $i$ 's such that $m_{i}=d$ in (1.2).

Theorem 1.9 ([5, Theorem 5.3], [12, Theorem 5.12]). Let $\mathcal{S}$ be a collection of points of $\mathbb{P}^{n}$ in general position. Let $D_{(r)}$ be as in Notation 1.6. Assume that

$$
\begin{aligned}
& 0 \leq m_{i} \forall i \in\{1, \ldots, s\}, \\
& m_{i}+m_{j} \leq d+1, \forall i, j \in\{1, \ldots, s\}, i \neq j(\text { if } s \geq 2) \\
& \sum_{i=1}^{s} m_{i} \leq n d+\left\{\begin{array}{ll}
n & \text { if } s \leq n+1 \text { and } d \geq 2 \\
1 & \text { if } s \leq n+1 \text { and } d=1 \\
1 & \text { if } s=n+2 \\
\min \{n-s(d), s-n-2\} & \text { if } s \geq n+3
\end{array} .\right.
\end{aligned}
$$

Then $h^{i}\left(D_{(r)}\right)=0$, for every $i \neq 0, r+1$. Moreover, $h^{i}(\tilde{D})=0$ for every $i \geq 1$.

Theorem 1.9 states that if $D$ is a special divisor on $X_{s,(0)}$, i.e. one for which the dimension of the first cohomology group does not vanish, then as long as it satisfies the bounds on the coefficients (1.5), its strict transform $\tilde{D}$ obtained after resolving the linear base locus is no more special, i.e. it has vanishing higher cohomology 
groups. In [5] the following question was posed, namely whether a similar statement is true for all cycles - linear and not linear- of $\mathrm{Bs}(|D|)$.

Question 1.10 ([5, Question 1.1]). Consider any effective divisor $D$ in the blownup $\mathbb{P}^{n}$ at general points. Let $\widetilde{X}$ be the smooth composition of blow-ups of $\mathbb{P}^{n}$ along the (strict transforms of the) cycles of the base locus of $|D|$, ordered in increasing dimension. Denote by $\widetilde{\mathcal{D}}$ the strict transform of the general divisor of $\mathcal{L}$ in $\widetilde{X}$. Does $h^{i}\left(\widetilde{X}, \mathcal{O}_{\widetilde{X}}(\widetilde{\mathcal{D}})\right)$ vanish for all $i \geq 1$ ?

Notice that we adopt the notation $\tilde{D}$ for the strict transform after the blowup of the linear base locus and $\widetilde{\mathcal{D}}$ for the strict transform after the blow-up of the whole base locus. An affirmative answer to Question 1.10 would imply that $h^{0}(D)=\chi\left(\widetilde{X}, \mathcal{O}_{\widetilde{X}}(\widetilde{\mathcal{D}})\right)$, the Euler characteristic. This would give the dimension of the linear system $\mathcal{L}$, answering the corresponding interpolation problem.

We also pose the following slightly different question.

Question 1.11. Let $X$ be the blown-up $\mathbb{P}^{n}$ at general points and let $D$ be an effective divisor on $X$. Let $(\widetilde{X}, \widetilde{D})$ be obtained via a log resolution of the pair $(X, D)$. Does $h^{i}\left(\widetilde{X}, \mathcal{O}_{\tilde{X}}(\widetilde{\mathcal{D}})\right)$ vanish for all $i \geq 1$ ?

In Section 2 for $s \leq n+2$ and $D$ effective, or for $s \geq n+3$ and $D$ effective having only linear base locus, Theorem 2.9 establishes that $\widetilde{\mathcal{D}}$ and $\tilde{D}$ coincide, answering positively Question 1.10 and Question 1.11.

\section{Globally generated divisors on $X_{s,(r)}$}

Recall that $D$ denotes a divisor on $X_{s,(0)}$ of the form (1.2) and $D_{(r)}$, as in (1.4), is its strict transform in $X_{s,(r)}$. See Notations 1.3, 1.6 and 1.8. If $\bar{r}$ is the dimension of the linear base locus of $D$, then we set $\tilde{D}=D_{(\bar{r})}$.

In this section we establish the following result, that classifies globally generated divisors of the form $D_{(r)}$, for every $r \geq 0$.

For any $s \geq n+3$ we introduce the following integer (see also [13, Theorem 2.2]):

$$
b_{0}:= \begin{cases}\min \{n-1, s-n-2\}-1 & \text { if } m_{1}=d-1 \text { and } m_{i}=1, i \geq 2 \\ \min \{n, s-n-2\}-1 & \text { otherwise, }\end{cases}
$$

while for $s \leq n+2$, we define $b_{0}:=-1$

Theorem 2.1. Assume that $\mathcal{S} \subset \mathbb{P}^{n}$ is a collection of points in general position. Assume that $s \leq n+1$ or that $s \geq n+2$ and $d$ is large enough, namely

$$
\sum_{i=1}^{s} m_{i}-n d \leq b_{0}
$$

Then for any $0 \leq r \leq n-1$ the divisor $D_{(r)}$ on $X_{s,(r)}$ is globally generated if and only if

$$
\begin{aligned}
& 0 \leq m_{i} \leq d, \forall i \in\{1, \ldots, s\}, \\
& 0 \leq(r+1) d-\sum_{i \in I} m_{i}, \forall I \subseteq\{1, \ldots, s\},|I|=r+2 .
\end{aligned}
$$

Remark 2.2. Notice that if $s \leq\left\lfloor\frac{r+2}{r+1} n\right\rfloor$, then the condition on the degree (2.2) is always satisfied. Hence in this range, Theorem 2.1 provides a complete classification of divisors $D$ on $X_{s,(0)}$ whose strict transform $D_{(r)}$ in $X_{s,(0)}$ is globally generated. 
Remark 2.3. If $\bar{r}=n-1$, then $\tilde{D}$ is the strict transform of $D_{(n-2)}$ obtained by subtraction of strict transforms of hyperplanes $L_{I(n)} \subset \mathbb{P}^{n}, X_{s,(n-2)}-\rightarrow X_{s,(n-2)}$, cfr. Remark 1.7. Therefore in the same assumptions of Theorem 2.1, we have that $\tilde{D}$ is globally generated if and only if

$$
\begin{aligned}
& 0 \leq m_{i} \leq d, \forall i \in\{1, \ldots, s\}, \\
& 0 \leq n d-\sum_{i \in I} m_{i}, \forall I \subseteq\{1, \ldots, s\},|I|=n+1 .
\end{aligned}
$$

In order to prove the main result of this section, Theorem 2.1, we need the following result.

Lemma 2.4. In the above notation, assume

$$
\sum_{i=1}^{s} m_{i} \leq n d+ \begin{cases}0 & \text { if } s \leq n+2 \\ \min \{n-s(d), s-n-2\} & \text { if } s \geq n+3 .\end{cases}
$$

Then for every exceptional divisor $E_{I}$ and any infinitely near point $q \in E_{I}$, we have

$$
h^{1}\left(D_{(r)} \otimes \mathcal{I}_{q}\right) \leq h^{1}\left(D_{(r)}-E_{I}\right) .
$$

Proof. We prove the statement by induction on $n$. We refer to [13, Theorem 2.11] for the proof of the statement for $n=2$. We will assume $n \geq 3$. Consider the short exact sequence

$$
0 \rightarrow D_{(r)}-\left.E_{I} \rightarrow D_{(r)} \otimes \mathcal{I}_{q} \rightarrow D_{(r)} \otimes \mathcal{I}_{q}\right|_{E_{I}} \rightarrow 0 .
$$

We claim that

$$
h^{1}\left(\left.D_{(r)} \otimes \mathcal{I}_{q}\right|_{E_{I}}\right)=0 .
$$

The statement will follow from this, by looking at the long exact sequence in cohomology associated with (2.6).

We now prove the claim, namely that (2.7) holds. In order to do this, we set $\rho+1:=|I|$ and we introduce the positive integer

$$
\alpha(I):=\left|\left\{I(\rho+1): k_{I(\rho+1)} \geq 1, I(\rho+1) \supset I\right\}\right|,
$$

where $I(\rho+1)$ denotes an index set contained in $\{1, \ldots, s\}$ of cardinality $\rho+2$ and the integer $k_{I(\rho+1)}$ is defined as in (5.2).

We recall that $E_{I}$ is a product, whose second factor is isomorphic to $X_{\alpha(I),(r-\rho-1)}^{n-\rho-1}$, the blown-up projective space of dimension $n-\rho-1$ along linear cycles up to dimension $r-\rho-1$ spanned by $\alpha(I)$ points. We refer to [12, Lemma $2.5]$ for details. Moreover, let $F$ be a divisor on the blow-up of $\mathbb{P}^{n-\rho-1}$ at $\alpha(I)$ points in linearly general position, $X_{\alpha(I),(0)}^{n-\rho-1}$, of the following form:

$$
F:=k_{I} h-\sum_{\substack{I(\rho+1) \supset I \\ k_{I(\rho+1)} \geq 1}} k_{I(\rho+1)} \cdot e_{I(\rho+1) \mid I} .
$$

We claim that the following holds.

(1) The restriction of $D_{(r)}$ to $E_{I}$ is $\left.D_{(r)}\right|_{E_{I}}=\left(0, F_{(r-\rho-1)}\right)$, where $F_{(r-\rho-1)}$ denotes the strict transform of $F$ in $X_{\alpha(I),(r-\rho-1)}^{n-\rho-1}$.

(2) If $D$ satisfies the bound (2.5), so does $F$. 
The proof of (2.7) follows from these two claims. Indeed (1) implies that

$$
\left.\mathcal{O}_{X_{(r)}}\left(D_{(r)}\right) \otimes \mathcal{I}_{q}\right|_{E_{I}} \cong \mathcal{O}_{X_{\alpha(I),(r-\rho-1)}^{n-\rho-1}}\left(F_{(r-\rho-1)}\right) \otimes \mathcal{I}_{q_{b}}
$$

where $q=\left(q_{b}, q_{f}\right) \in E_{I}$. This has vanishing first cohomology group, by induction on $n$, since $F$ satisfies (2.5), by (2).

We first prove (1). The fact that the first factor of the restriction $D_{(r)} \mid E_{I}$ is zero follows from the computation of normal bundles of the $E_{I}$ 's and from intersection theory on $X_{s,(r)}$, see [5, Section 4] or [12, Section 2]. We now compute the second factor. We have

$$
\begin{aligned}
\left.E_{I}\right|_{E_{I}} & =(*, h) \\
\left.E_{I}\right|_{E_{I(j)}} & =\left(*, e_{J \mid I}\right), \text { for all } J \supset I,
\end{aligned}
$$

where $*$ denotes the appropriate divisor on the first factor; here we are only interested in the second factor. The classes of the divisors $h$ and $e_{J \mid I}$ generate the Picard group of $X_{\alpha(I),(r-\rho-1)}^{n-\rho-1}$. We can compute

$$
\left.D_{(r)}\right|_{E_{I}}=\left(0, k_{I} h-\sum_{J \supset I} k_{J} e_{J \mid I}\right) .
$$

To conclude, we need to prove that the second factor on the right hand side of the above expression equals the strict transform $F_{(r-i-1)}$. Notice first of all that for any integer $\tau \geq 1$, one has $|I(\tau+\rho+1) \backslash I|=\tau+1$. Hence it is enough to prove that

$$
\sum_{\substack{(\rho+1) \supset I: \\ I(\rho+1) \subset I(\tau+\rho+1)}} k_{I(\rho+1)}-\tau k_{I}=k_{I(\tau+\rho+1)} .
$$

By definition, the left hand side equals

$$
\begin{aligned}
& \sum_{I \subset I(\rho+1) \subset I(\tau+\rho+1)} k_{I}+\sum_{j \in I(\tau+\rho+1) \backslash I} m_{j}-(\tau+1) d-\tau k_{I}= \\
& =(1+\tau) k_{I}+\sum_{j \in I(\tau+\rho+1) \backslash I} m_{j}-(\tau+1) d-\tau k_{I} \\
& =k_{I}+\sum_{j \in I(\tau+\rho+1) \backslash I} m_{j}-(\tau+1) d \\
& =k_{I(\tau+\rho+1)} .
\end{aligned}
$$

We now prove (2). We shall assume that $m_{i} \geq 1$ for all $i \in \mathcal{S}$. Notice that for any index set $I(\rho+1)=I \cup\{j\}$, with $k_{I \cup\{j\}} \geq 1$, we can write

$$
k_{I(\rho+1)}-k_{I}=m_{j}-d .
$$

Set $A(I)=\left\{j \in S \backslash I: k_{I \cup\{j\}} \geq 1\right\}$. We have

$$
\alpha:=|A(I)|=\alpha(I) \leq s-\rho-1 .
$$


We denote by $s_{F}\left(k_{I}\right)$ the number of sets $I(\rho+1)=I \cup\{j\}$ such that $k_{I(\rho+1)}=k_{I}$. The divisor $F$ satisfies (2.5) if and only if

$$
\begin{aligned}
\sum_{j \in A(I)} k_{I \cup\{j\}} \leq & (n-\rho-1) k_{I} \\
& + \begin{cases}0 & \text { if } \alpha \leq n-\rho+1, \\
\min \left\{n-\rho-1-s_{F}\left(k_{I}\right), \alpha-n+\rho-1\right\} & \text { if } \alpha \geq n-\rho+2 .\end{cases}
\end{aligned}
$$

Using (2.10), we can see that (2.12) is equivalent to

$$
\begin{aligned}
\sum_{j \in A(I)} m_{j}-\alpha d \leq & (n-\rho-1-\alpha) k_{I} \\
& + \begin{cases}0 & \text { if } \alpha \leq n-\rho+1, \\
\min \left\{n-\rho-1-s_{F}\left(k_{I}\right), \alpha-n+\rho-1\right\} & \text { if } \alpha \geq n-\rho+2 .\end{cases}
\end{aligned}
$$

For $\alpha \leq n-\rho-1$, this holds since $\alpha=|A(I)|$ and $m_{j} \leq d$ so that the left hand side of (2.13) is a non-positive integer, while the right hand side is non-negative.

For $\alpha \geq n-\rho$, the left hand side of (2.13) equals

$$
\sum_{j \in S} m_{j}-n d-k_{I}-\sum_{j \in S \backslash(I \cup A(I))} m_{j}-(\alpha-n+\rho) d,
$$

therefore (2.13) is equivalent to

$$
\begin{aligned}
& (\alpha-n+\rho)\left(k_{I}-d\right)-\sum_{j \in S \backslash(I \cup A(I))} m_{j}+\left(\sum_{j \in S} m_{j}-n d\right) \\
& \leq \begin{cases}0 & \text { if } \alpha \leq n-\rho+1, \\
\min \left\{n-\rho-1-s_{F}\left(k_{I}\right), \alpha-n+\rho-1\right\} & \text { if } \alpha \geq n-\rho+2 .\end{cases}
\end{aligned}
$$

Notice that $|S \backslash(I \cup A(I))|=s-\rho-1-\alpha$. Using this, $k_{I}-d \leq 0$ and $m_{i} \geq 1$, we obtain that the left hand side of (2.14) is bounded above by

$$
-(s-\rho-1-\alpha)+ \begin{cases}0 & \text { if } s \leq n+2, \\ \min \{n-s(d), s-n-2\} & \text { if } s \geq n+3 .\end{cases}
$$

To conclude, it is enough to show that (2.15) is bounded above by the expression in the right hand side of (2.14). We do so in the following cases.

Case (a) $s \leq n+2$. The inequality (2.11) implies that $\alpha \leq n-\rho+1$. The right hand side of (2.14) is zero. Moreover (2.15) equals $-(s-\rho-1-\alpha)$ which is non-positive, therefore we conclude in this case.

Case (b) $s \geq n+3$ and $\alpha \leq n-\rho+1$. As above the right hand side of (2.14) is zero. Moreover (2.15) is bounded above by $-(s-\rho-1-\alpha)+s-n-2$ which is non-positive, so we conclude.

Case (c) $s \geq n+3$ and $\alpha \geq n-\rho+2$. We need to prove that (2.16) $-(s-\rho-1-\alpha)+\min \{n-s(d), s-n-2\} \leq \min \left\{n-\rho-1-s_{F}\left(k_{I}\right), \alpha-n+\rho-1\right\}$.

Case (c.1) Assume that $k_{I}=d$. Notice that $m_{i}=d$ for all $i \in I$ and $s_{F}\left(k_{I}\right)$ corresponds to the cardinality of the set $\left\{i \in \mathcal{S} \backslash I: m_{i}=d\right\}$. Therefore $s(d)=$ $s_{F}\left(k_{I}\right)+\rho+1$. Moreover, using $m_{i} \geq 1$ we also obtain $\alpha=s-\rho-1$. Therefore $\min \{n-s(d), s-n-2\}=\min \left\{(n-\rho-1)-s_{F}\left(k_{I}\right), \alpha-n+\rho-1\right\}$ and this concludes the proof. 
Case (c.2) Assume that $k_{I}-d \leq-1$. Since $\alpha-n+\rho \geq 2$ then the left hand side of (2.14) is bounded above by

$$
\begin{aligned}
-(\alpha-n+\rho)-(s-\rho-1-\alpha)+\min \{n-s(d), s-n-2\} & \leq \\
-(\alpha-n+\rho)-(s-\rho-1-\alpha)+(s-n-2) & =-1 .
\end{aligned}
$$

Therefore we conclude because the right hand side of (2.14) is positive by definition.

Proof of Theorem 2.1. The case $r=0$ is proved in [13, Corollary 2.4]. We will assume $r \geq 1$. In order to prove that $D_{(r)}$ is globally generated, we will prove that $h^{1}\left(D_{(r)} \otimes \mathcal{I}_{q}\right)=0$, for all points $q \in X_{s,(r)}$. We distinguish the following two cases:

(1) $q$ lies on some exceptional divisor $E_{I}$,

(2) $q$ is the pull-back of a point outside of the union $\sum_{I \subseteq\{1, \ldots, s\},|I| \leq r+1} L_{I} \subset \mathbb{P}^{n}$.

Case (1). Assume $q \in E_{I}$ and write $\rho+1:=|I|, \rho \leq r$. We want to prove that $h^{1}\left(D_{(r)}-E_{I}\right)=0$. Then the conclusion will follow from Theorem 2.4.

Reordering the points if necessary, we may assume that $1 \in I$. Define the divisor $D^{\prime}=D-E_{1}$ in $X_{s,(0)}$. One can easily check that $D^{\prime}$ satisfies the hypotheses of Theorem 1.9, therefore $h^{1}\left(D_{(r)}^{\prime}\right)=0$.

Consider the set $\mathcal{J}$ of all indices $J$ of cardinality $1 \leq|J| \leq r+1$ such that $1 \in J$ and such that $\sum_{i \in J} m_{i}-|J| d \geq 0$. We consider on $\mathcal{J}$ the graded lexicographical order, namely if $\left|J_{1}\right|<\left|J_{2}\right|$ then $J_{1} \prec J_{2}$, while if $\left|J_{1}\right|=\left|J_{2}\right|$ we use the lexicographical order.

Notice that $I \in \mathcal{J}$ and that $D_{(r)}^{\prime}=D_{(r)}-\sum_{J \in \mathcal{J}} E_{J}$. In other words,

$$
D_{(r)}-E_{I}=D_{(r)}^{\prime}+\sum_{\substack{J \in \mathcal{J}: \\ J \neq I}} E_{J}
$$

We can obtain $D_{(r)}^{\prime}$ as the residual of iterative applications of short exact sequences starting from $D_{(r)}^{\prime}+\sum_{J \in \mathcal{J}, J \neq I} E_{J}$ by restrictions to the exceptional divisors $E_{J}$, with $J \in \mathcal{J}, J \neq I$, following the order on $\mathcal{J}$. More precisely, we start from $D_{(r)}^{\prime}+\sum_{J \in \mathcal{J}, J \neq I} E_{J}$ and we restrict it to $E_{1}$, then we take the kernel of the so obtained exact sequence and we restrict, iteratively, to all $E_{J}$ 's, with $J \in \mathcal{J} \backslash\{I\}$, $|J|=2$, then to all $E_{J}$ 's, with $J \in \mathcal{J} \backslash\{I\},|J|=3$, etc. We claim that each restricted divisor has vanishing first cohomology group. This gives a proof of the statement, since the last kernel, that is $D_{(r)}^{\prime}$, has vanishing first cohomology too, by Theorem 1.9.

To prove the claim, recall that each exceptional divisor $E_{J}$ is the product of two blown-up projective spaces of dimension $|J|-1$ and $n-|J|$ respectively, see [12, Lemma 2.6]. In particular the first component is isomorphic to $\mathbb{P}^{|J|-1}$ blown-up along linear cycles (see Remark 1.5). Let us denote by $h$ the class of a general hyperplane on the first component and by $e_{J^{\prime}}$ the class of the restriction of the exceptional divisors $E_{J^{\prime}}$, namely $\left.E_{J^{\prime}}\right|_{E_{J}}=\left(e_{J^{\prime}}, 0\right)$, for all $J^{\prime} \subset J$. Let $\operatorname{Cr}(h)$ be the proper transform of the standard Cremona transformation of the hyperplane class $h$ on $\mathbb{P}^{|J|-1}$, i.e.

$$
\operatorname{Cr}(h)=(|J|-1) h-\sum_{\substack{J^{\prime} \subset J: \\\left|J^{\prime}\right|<|J|-1}}\left(|J|-\left|J^{\prime}\right|-1\right) e_{J^{\prime}} .
$$


We have

$$
\begin{aligned}
\left.D_{(r)}^{\prime}\right|_{E_{J}} & =(0, *) \text { for every } E_{J}, \\
\left.E_{J}\right|_{E_{J}} & =(-\operatorname{Cr}(h), *), \\
\left.E_{J^{\prime}}\right|_{E_{J}} & =(0, *) \text { for all } J^{\prime} \supset J,
\end{aligned}
$$

where we use $*$ to denote the appropriate divisor on the second factor. See $[12$, Sect. 2,3] for details.

Therefore each of the above restrictions is

$$
\left.\left(D_{(r)}^{\prime}+\sum_{\substack{J^{\prime} \in \mathcal{J}: \\ J^{\prime} \neq I, J \prec J^{\prime}}} E_{J^{\prime}}\right)\right|_{E_{J}}=(-\operatorname{Cr}(h), *) .
$$

It has vanishing first cohomology group by [12, Theorem 3.1]. This concludes the proof of Case (1).

Case (2). In this case $q$ is the pull-back of a point $q^{\prime} \in \mathbb{P}^{n} \backslash \bigcup_{I \subset\{1, \ldots, s\},|I| \leq r} L_{I}$. We prove the claim by induction on $n$. The case $n=1$ is obvious. Assume $n \geq 2$. We distinguish two subcases.

Case (2.a). Let us assume first that the points $p_{1}, \ldots, p_{s}, q^{\prime}$ are not in linearly general position. If $s \geq n, q^{\prime}$ lies on a hyperplane $H$ of $\mathbb{P}^{n}$ spanned by $n$ points of $\mathcal{S}$. Reordering the points if necessary, assume that $q^{\prime} \in H:=\left\langle p_{1}, \ldots, p_{n}\right\rangle$. If $s<n$, let $H$ be any hyperplane containing $\mathcal{S} \cup\left\{q^{\prime}\right\}$. Let $\bar{H}$ denote the pull-back of $H$ on $X_{s,(r)}$. It is isomorphic to the space $\mathbb{P}^{n-1}$ blow-up along linear cycles of dimension up to $\min \{r, n-2\}$, spanned by $\bar{s}:=\min \{s, n\}$ points in general position, that we may denote by $\bar{H} \cong X_{\bar{s},(r)}^{n-1}$ as in Remark 1.5, part (1). As a divisor, we have

$$
\bar{H}=H-\sum_{i=1}^{\bar{s}-1} E_{i}-\sum_{\substack{I \subset\{1, \ldots, \bar{s}\}:, 1 \leq|I| \leq \min \{r, n-2\}}} E_{I} .
$$

Consider the restriction exact sequence of line bundles

$$
0 \rightarrow D_{(r)}-\left.\bar{H} \rightarrow D_{(r)} \otimes \mathcal{I}_{q} \rightarrow\left(D_{(r)} \otimes \mathcal{I}_{q}\right)\right|_{\bar{H}} \rightarrow 0 .
$$

The restriction, $\left.D_{(r)}\right|_{\bar{H}} \otimes \mathcal{I}_{q}$, is a toric divisor on the blown-up space $\bar{H} \cong X_{\bar{s},(r)}^{n-1}$, with a point, $q$, in possible special linear configuration with the other points (see [12, Section 5.3] for a short introduction on toric divisors on blown-up projective spaces). We conclude that it has vanishing first cohomology group by induction on $n$. The kernel also has vanishing first cohomology because its base locus possibly contains linear cycles with multiplicity one, see [12, Theorem 1.5]. We conclude that $h^{1}\left(D_{(r)} \otimes \mathcal{I}_{q}\right)=0$.

Case (2.b). Let us assume now that the points $p_{1}, \ldots, p_{s}, q^{\prime}$ are in linearly general position. If $s \geq n-1$, let $H$ denote the hyperplane $\left\langle p_{1}, \ldots, p_{n-1}, q^{\prime}\right\rangle$. If $s<n-1$, let $H$ be any hyperplane containing $\mathcal{S} \cup\left\{q^{\prime}\right\}$. In both cases such an $H$ exists by the assumption that $\mathcal{S}$ is a set of points in general position. Let $\bar{H}$ denote the pull-back of $H$ on $X_{s,(r)}$, as in (2.18) and consider the corresponding restriction sequence as in (2.19). 
As in Case (2.a), we conclude by induction on $n$ and by noticing that the kernel has only possibly simple linear obstructions.

Assume now that one of the inequalities in (2.3) does not hold. We claim that $D_{(r)}$ is not globally generated. Indeed, if $m_{i} \geq d+1$ then the divisor $D$ is not effective therefore $D_{(r)}$ is not globally generated. If $m_{i} \leq-1$ then the divisor $E_{i}$ is in the base locus of $D_{(r)}$. If $k_{I} \geq 1$ for some $I$ such that $|I|=r+2$ and $r \leq n-1$, then the divisor $D_{(r)}$ contains in its base locus the strict transform of the linear cycle $L_{I}$ by Lemma 5.2 , therefore is not globally generated.

Remark 2.5. The strict transform $\tilde{D}=D_{(\bar{r})}$ of $\mathcal{L}$ is base point free if (2.3) is satisfied with $r=\bar{r}$.

2.1. Vanishing cohomology of strict transforms. In this section we will determine the base locus and their intersection multiplicity for divisors satisfying condition (2.5). Furthermore, Theorem 2.9 answers Question 1.1 posed in [5] in this range.

Recall that a line bundle is globally generated if and only if the associated linear system is base point free. We are now ready to prove that Theorem 2.1 implies a complete description of the base locus of all non-empty linear systems in $\mathbb{P}^{n}$ of the form $\mathcal{L}=\mathcal{L}_{n, d}\left(m_{1}, \ldots, m_{s}\right)$ (1.1) that are only linearly obstructed.

Theorem 2.6 (Base locus of linear systems). The base locus of the divisor $D_{(r)}$ on $X_{s,(r)}$, strict transform of $D=d H-\sum_{i=1}^{s} m_{i} E_{i}$ satisfying (2.2), namely

$$
\sum_{i=1}^{s} m_{i} \leq n d+b_{0}
$$

is the formal sum

$$
\sum_{\substack{I \subseteq\{1, \ldots, s\}: \\|I| \geq r+1}} k_{I} L_{I} \in A^{*}\left(X_{s,(r)}\right) .
$$

In particular, if $s \leq n+2$, the sum (2.20) with $r=-1$, describes the base locus of all non-empty linear systems $\mathcal{L}_{n, d}\left(m_{1}, \ldots, m_{s}\right)$, while if $s \geq n+3$ the sum $(2.20)$ with $r=-1$, describes the base locus of non-empty linear systems satisfying the bound (2.2).

Proof. By [6, Lemma 4.1] (cf. also Lemma 5.2 below), each linear subspace $L_{I}$ with $k_{I}>0$ is a base locus cycle for $\mathcal{L}$ and $k_{I}$ is its exact multiplicity of containment. Therefore

$$
\bigcup_{\substack{I \subseteq\{1, \ldots, s\}: \\|I| \geq r+1}} k_{I} L_{I} \subset \operatorname{Bs}\left(\left|D_{(r)}\right|\right) .
$$

By Theorem 2.1, the strict transform $D_{(r)}$ of an element of $\mathcal{L}$ is base point free as soon as no higher dimensional cycle, i.e. no $(r+1)$-plane, is contained in the base locus, namely when $k_{I} \leq 0$ for all $I \subseteq\{1, \ldots, s\}$ of cardinality $r+1$. In particular, if $\bar{r}$ is the dimension of the linear base locus of $D$, then $\tilde{D}=D_{(\bar{r})}$ is base point free. 
Since the total transform of $D_{(r)}$ in $X_{s,(\bar{r})}$ equals

$$
\tilde{D}+\sum_{\substack{I \subseteq\{1, \ldots, s\}: \\|I| \geq r+1}} k_{I} E_{I},
$$

one concludes that the base locus of $D$ is supported only along linear cycles

$$
\operatorname{Bs}\left(\left|D_{(r)}\right|\right) \subset \bigcup_{\substack{I \subseteq\{1, \ldots, s\}: \\|I| \geq r+1}} k_{I} L_{I} .
$$

Remark 2.7. The unique rational normal curve $C$ of degree $n$ through $n+3$ points of $\mathbb{P}^{n}$ and its secant varieties $\sigma_{t}(C)$ were studied in [6] as cycles of the base locus of non-empty linear systems $\mathcal{L}$. By [6, Lemma 4.1], condition (2.2) for $s=n+3$ says that neither $C$ nor $\sigma_{t}(C)$ are contained in the base locus of $\mathcal{L}$ (cf. also Lemma 5.2 below). Hence Theorem 2.6 states that if $C$ is not in the base locus of $\mathcal{L}$, then nothing else is, besides the linear cycles.

Remark 2.8. Recall that for a line bundle $D_{(r)}$ as above, the stable base locus is defined as

$$
\mathbb{B}\left(D_{(r)}\right)=\cap_{m \in \mathbb{N}} \operatorname{Bs}\left(\left|m D_{(r)}\right|\right) .
$$

The obvious equality $k_{I}(m D)=m k_{I}(D)$, for any integer $m \geq 1$, and Theorem 2.6 show that $\operatorname{Bs}\left(\left|m D_{(r)}\right|\right)=m \cdot \operatorname{Bs}\left(\left|D_{(r)}\right|\right)$. Therefore the base locus of $D_{(r)}$ is stable, namely

$$
\mathbb{B}\left(D_{(r)}\right)=\operatorname{Bs}\left(\left|D_{(r)}\right|\right) .
$$

Because the stable base locus is invariant under taking multiples, we can extend this definition to the case of $\mathbb{R}$-divisors, see [4, Lemma 3.5.3]. In particular we can consider the stable base locus of $\epsilon D_{(r)}$, for any $\epsilon \in \mathbb{R}$, and obtain

$$
\mathbb{B}\left(\epsilon D_{(r)}\right)=\mathbb{B}\left(D_{(r)}\right)=\operatorname{Bs}\left(\left|D_{(r)}\right|\right) .
$$

We now can prove that the strict transform of $D$ in the iterated blow-up along its base locus has vanishing cohomology groups. This answers affirmatively Question 1.10 .

Theorem 2.9. Let $\mathcal{S}$ be a collection of points in general position and $D$ be any effective divisor on $X_{s,(0)}$. Assume the following holds:

$$
\sum_{i=1}^{s} m_{i}-n d \leq \begin{cases}0 & \text { if } s \leq n+2 \\ \min \{n-s(d), s-n-2\} & \text { if } s \geq n+3\end{cases}
$$

Then Question 1.10 has affirmative answer.

Proof. Since all cycles of the base locus of the divisors $D$ are linear by Theorem 2.6, we conclude that $\widetilde{\mathcal{D}}$ equals $\tilde{D}$. The claims follow from Theorem 1.9. 
2.2. Log resolutions for divisors on blown-up projective spaces in points. Let $D$ be any divisor on $X_{s,(0)}$ in the hypothesis of Theorem 2.9 and let $\bar{r}$ be the maximum dimension of its linear base locus. If the divisor $D$ has $\bar{r}<n-1$, Theorem 2.9 implies that the map $X_{s,(\bar{r})} \rightarrow X_{s,(0)}$ is a resolution of singularities of $D$.

Moreover, we recall that a log resolution of the pair $\left(X_{s,(0)}, D\right)$ is a birational morphism $\pi: Y \rightarrow X_{s,(0)}$ such that the pair $(Y, \tilde{D})$ is $\log$ smooth, where $\tilde{D}=\pi_{*}^{-1} D$ is the strict transform of $D$, namely such that $Y$ is smooth and the sum $\tilde{D}+\operatorname{Exc}(\pi)$, where $\operatorname{Exc}(\pi)$ is the sum of exceptional divisors of $\pi$, is simple normal crossing. For $D$ a general divisor of the linear system $\left|d H-\sum_{i=1}^{s} m_{i} E_{i}\right|$ as in Notation 1.3, we obtain the following result.

Corollary 2.10. If $D$ satisfies relation (2.2), then the pair $\left(X_{s,(\bar{r})}, D_{(\bar{r})}\right)$ is $\log$ smooth and the birational morphism $X_{s,(\bar{r})} \rightarrow X_{s,(0)}$ is a $\log$ resolution of the singularities of the pair $\left(X_{s,(0)}, D\right)$.

Proof. The variety $X_{s,(\bar{r})}$ is smooth. Indeed at each step of the blow-up of $X$, $X_{s,(r)} \rightarrow X_{s,(r-1)}, r \leq \bar{r}$, the center of the blow-up is a disjoint union of smooth subvarieties, namely the strict transforms of the liner cycles $L_{I(r)}$ with $k_{I(r)}>$ 0 (see [12, Subsection 4.2] for details). This, together with the fact that each exceptional divisor is smooth, also proves that the $\operatorname{sum} \operatorname{Exc}(\pi)=\sum_{r=1}^{n-2} \sum_{I(r)} E_{I(r)}$ is simple normal crossing. Moreover $D_{(\bar{r})}$ is base point free and its support intersects transversally all exceptional divisors $E_{I(r)}$, by Theorem 2.6. Finally since $D$ is general, then $D_{(\bar{r})}$ is general too hence it is smooth by Bertini's theorem.

\section{LOG ABUNDANCE FOR LINEARLY OBSTRUCTED DIVISORS ON $X_{s,(0)}$}

In this section we construct an infinite family of log canonical pairs given by effective divisors on the blow-up of $\mathbb{P}^{n}$ at $s$ points in general position, $X_{s,(0)}$, with arbitrary $s$. Moreover we prove that the $(\log )$ abundance conjecture holds for these pairs, when $D$ is only linearly obstructed, namely $D$ has only linear base locus.

In the case $s \leq n+3$, the blown-up spaces $X_{s,(0)}$ are Mori dream spaces (see $[2,9,29]$ for an explicit proof), therefore it is a well-known fact that all nef divisors are semi-ample. Moreover this statement holds for $s \leq 2 n$, see [13, Theorem 3.2] and [2, Proposition 1.4]. For $s \geq n+4$, the result is new (Section 3.1).

Notation 3.1. ([25]) Let $(X, \Delta)$ be a $\log$ pair, with $X$ a normal variety and $\Delta=$ $\sum_{j} a_{j} \Delta_{j}$ a formal $\mathbb{Q}$-linear combination of prime divisors. Let $\pi: Y \rightarrow X$ be a log resolution of $(X, \Delta)$, denote by $\tilde{\Delta}:=\pi_{*}^{-1} \Delta$ the strict transform of $\Delta$ and by $E_{i}$ the exceptional divisors. Write

$$
K_{Y}+\tilde{\Delta}=\pi^{*}\left(K_{X}+\Delta\right)+\sum_{i} a\left(E_{i}, X, \Delta\right) E_{i},
$$

with $a\left(E_{i}, X, \Delta\right) \in \mathbb{Q}$. If $a_{j} \leq 1$, then the discrepancy of the pair $(X, \Delta)$ can be computed as

$$
\operatorname{discrep}(X, \Delta)=\min _{i}\left\{a\left(E_{i}, X, \Delta\right), \min _{j}\left\{1-a_{j}\right\}, 1\right\},
$$

see [25, Corollary 2.32]. The pair $(X, \Delta)$ is said to be $\log$ canonical $(l c)$ if $\operatorname{discrep}(X, \Delta) \geq-1$. 
Conjecture 3.2 ([25, Conjecture 3.12]). Let $(X, \Delta)$ be lc, $\Delta$ effective. Then $K_{X}+$ $\Delta$ is nef if and only if it is semi-ample.

As an application of the results of this paper, we prove that the log abundance conjecture holds for $\left(X_{s,(0)}, \Delta\right)$, where $\Delta:=\epsilon D$ with $0 \leq \epsilon \leq 1$ and $D \geq 0$ effective divisor on $X_{s,(0)}$, by explicitly constructing a $\log$ resolution of the pair.

We recall here that if $s \leq n+1$ or $s \geq n+2$ and (2.2) holds, the divisor $D$ is effective, by [5, Theorem 5.3]. For a small number of points, $s \leq n+2$, or an arbitrary number of points with a bound on the coefficients of the divisors, we construct an infinite family of log canonical pairs given by effective divisors $D$ on $X_{s,(0)}$.

Theorem 3.3. Fix integers $n>3$, arbitrary s. Let $D=d H-\sum_{i=1}^{s} m_{i} E_{i}$ be a general effective divisor on $X=X_{s,(0)}$ with $s \leq n+2$ or with $s \geq n+3$ and satisfying (2.2). For every $\epsilon \in \mathbb{Q}^{\geq 0}$, such that

$$
\begin{aligned}
& \epsilon m_{i} \geq n-1, \forall i \in\{1, \ldots, s\} \\
& \epsilon\left(m_{i}+m_{j}-d\right) \leq n-3, \forall i, j \in\{1, \ldots, s\}, i \neq j,
\end{aligned}
$$

the pair $(X, \Delta)$ is lc.

Recall the classes of the canonical divisors on $X$,

$$
K_{X}=-(n+1) H+(n-1) \sum E_{i},
$$

and consider the $\mathbb{Q}$-divisor

$$
K_{X}+\Delta=(\epsilon d-n-1) H-\sum_{i}\left(\epsilon m_{i}-n+1\right) E_{i} .
$$

Corollary 3.4. In the same hypotheses of Theorem 3.3 then

(1) Conjecture 3.2 holds for the pair $(X, \Delta)$, namely if $K_{X}+\Delta$ is nef then it is semi-ample.

(2) The canonical ring

$$
\bigoplus_{l \geq 0} H^{0}\left(X, \mathcal{O}_{X}\left(l K_{X}+\lfloor l \Delta\rfloor\right)\right)
$$

is finitely generated.

Proof. By Theorem 3.3, the pair $(X, \Delta)$ is lc. To conclude the first part, it is easy to see that under the condition (2.2), then the divisor $K_{X}+\Delta$ is nef (equiv. semi-ample) if and only if the conditions (3.2) of Theorem 3.3 are verified. This follows from [13, Theorem 3.1]. In fact, if $s \leq 2 n$, the nef and the semi-ample cone coincide, and the thesis follows trivially. Otherwise, if $s \geq 2 n+1$, (2.2) implies that the hypotheses of [13, Theorem 3.1] are satisfied. The first statement follows.

For the second statement see [25, Section 3.13].

Remark 3.5. Let $0 \leq \epsilon \ll 1, \epsilon \in \mathbb{R}$. Let $\Delta=\epsilon D \in N^{1}\left(X_{s,(0)}\right)_{\mathbb{R}}$ be an $\mathbb{R}$-divisor on $X_{s,(0)}$ satisfying the assumption of Theorem 3.3. Then Conjecture 3.2 holds for the pair $(X, \Delta)$. This follows from Theorem 3.3 and Remark 2.8.

Remark 3.6. In the notation of Theorem 3.3, if $s \leq n+2$ or $s \geq n+3$ and condition (2.2) is satisfied, then $D$ is effective and only linearly obstructed, see [5, Theorem 5.3]. In this case a log resolution of the corresponding pair is given by the iterated blow-up of the linear base locus, see Corollary 2.10. 
The case $s=n+3$ is the first case where non-linear obstructions appear for divisors violating (2.2). The base locus of effective divisors $D$ was studied in [6] (cf. Lemma 5.2 below). In this case a log resolution of any pair given satisfying (3.2), will be constructed in Section 6.1 by means of the iterated blow-up along the subvarieties, linear and non-linear, contained in the base locus.

3.1. Proof of Theorem 3.3, only linearly obstructed case. Let $s$ be an arbitrary integer. Set $X:=X_{s,(0)}$ and $Y:=X_{s,(n-2)}$. Let

$$
D=d H-\sum_{i=1}^{s} m_{i} E_{i} \geq 0
$$

be a divisor as in Notation 1.3. Assume moreover that $D$ is only linearly obstructed, namely that condition (2.2) is verified.

Remark 3.7. Notice that under the assumption (3.2) the divisor $D$ is irreducible. We recall that $D$ represents a general member of the linear system $|D|$ and the assumptions above force $D$ to have no divisorial components. Indeed, observe that no (strict transform of) hyperplane spanned by $n$ points is contained in the base locus of $D$. In fact, we can see first of all that (3.2) implies that for every $i=1 \ldots s$,

$$
\epsilon\left(m_{i}-d\right) \leq-2
$$

Now, if $I=I(n-1)$ is an index set of cardinality $n$, we can compute that the multiplicity of containment of the corresponding hyperplane is zero as follows:

$$
\begin{aligned}
\sum_{i \in I} m_{i}-(n-1) d & =\left(\sum_{i \in I \backslash\left\{i_{1}, i_{2}\right\}} m_{i}-(n-2) d\right)+\left(m_{i_{1}}+m_{i_{2}}-d\right) \\
& \leq-2(n-2)+(n-3) \\
& \leq 0 .
\end{aligned}
$$

Using the notation introduced in Subsection 1.4, let $\pi: Y \rightarrow X$ be the composition of blow-ups of $X$ along lines, then planes etc., up to codimension-2 linear cycles $L_{I}, I \subset\{1, \ldots, s\}$ for which $k_{I}>0$, see (1.3) for the definition of $k_{I}$. As in Notation 1.6, the strict transform of $D$, is given by

$$
\tilde{D}=d H-\sum_{i} m_{i} E_{i}-\sum_{r=1}^{n-2} \sum_{I(r)} k_{I(r)} E_{I(r)} .
$$

Proposition 3.8. In the above notation, the map $\pi: Y \rightarrow X$ is a log resolution of $(X, \Delta)$, for every $\epsilon \geq 0$.

Proof. It follows from Corollary 2.10.

Recall the class of the canonical divisors on $Y$ :

$$
K_{Y}=-(n+1) H+(n-1) \sum E_{i}+\sum_{r=1}^{n-2}(n-r-1) \sum_{I(r)} E_{I(r)} .
$$

Here we abuse notation by denoting by $H$ the hyperplane class in both $X$ and $Y$; similarly by abuse of notation we denote by $E_{i}$ an exceptional divisor in $X$ and its strict transform in $Y$. 
For $0 \leq \epsilon<1$, consider the pairs $(X, \Delta)=\left(X_{s,(0)}, \epsilon D\right)$ and $(Y, \tilde{\Delta})=$ $\left(X_{s,(n-2)}, \epsilon \tilde{D}\right)$ and write

$$
K_{Y}+\tilde{\Delta}=\pi^{*}\left(K_{X}+\Delta\right)+\sum_{1 \leq r \leq n-2}\left(n-r-1-\epsilon k_{I(r)}\right) E_{I(r)} .
$$

We have

$$
a_{i}\left(E_{I(r)}, X, \Delta\right)=n-r-1-\epsilon k_{I(r)},
$$

for any $I(r)$ such that $1 \leq r \leq n-2$ (cf. [25, Lemma 2.29]). Therefore $\operatorname{discrep}(X, \Delta) \geq-1$ if

$$
\epsilon k_{I(r)} \leq n-r, \forall I(r), 1 \leq r \leq n-2 .
$$

We are now ready to prove the first main result of this section.

Proof of Theorem 3.3, only linearly obstructed case. We are now ready to prove Theorem 3.3 for divisors with only linear obstructions. By Proposition 3.8, $(Y, \tilde{\Delta})$ is $\log$ smooth and $\pi: Y \rightarrow X$ is a $\log$ resolution of $(X, \Delta)$. We are going to prove that the pair is $(X, \Delta)$ is lc.

We prove that (3.4) is implied by (3.2), second line, and by (3.3). Indeed, take for instance $r=2$. If $k_{I(2)}=0$ the statement is obvious. Assume that $k_{I(2)}>0$. Write $I(2)=\left\{i_{1}, i_{2}, i_{3}\right\}$. We have $\epsilon k_{I(2)}=\epsilon\left(\left(m_{i_{1}}-d\right)+\left(m_{i_{2}}+m_{i_{3}}-d\right)\right) \leq-2+(n-3) \leq$ $n-2$. The same holds for $r \geq 3$.

\section{ON THE F-CONJECTURE}

In this section we discuss an application of our results. Let $\overline{\mathcal{M}}_{0, n}$ be the moduli space of stable rational curves with $n$ marked points. For $n=5, \overline{\mathcal{M}}_{0, n}$ is a del Pezzo surface and it has the property of being a Mori dream space. Hu and Keel in [20] showed that $\overline{\mathcal{M}}_{0,6}$ is a log Fano threefold, hence a Mori dream space; Castravet computed its Cox ring in [8]. For $n \geq 10, \overline{\mathcal{M}}_{0, n}$ is known to not be a Mori dream space, see $[10,18,26]$.

We recall here the $F$-conjecture on the nef cone of $\overline{\mathcal{M}}_{0, n}$ proposed by Fulton. The elements of the 1-dimensional boundary strata on $\overline{\mathcal{M}}_{0, n}$ are called $F$-curves. A divisor intersecting non-negatively all F-curves is said to be $F$-nef. The F-Conjecture states that a divisor on $\overline{\mathcal{M}}_{0, n}$ is nef if and only if it is F-nef. This conjecture was proved for $n \leq 7$ in by Keel and McKernan [24].

4.1. Preliminaries and notation. Let $\mathcal{I}$ be a subset of $\{1, \ldots, n+3\}$ with cardinality $2 \leq|\mathcal{I}| \leq n+1$ and let $\Delta_{\mathcal{I}}$ denote a boundary divisor on $\overline{\mathcal{M}}_{0, n+3}$. Here, $\Delta_{\mathcal{I}}$ is the divisor parametrizing curves with one component marked by the elements of $\mathcal{I}$ and the other component marked by elements of its complement, $\mathcal{I}^{c}$, in $\{1, \ldots, n+3\}$. Obviously $\Delta_{\mathcal{I}}=\Delta_{\mathcal{I}^{c}}$.

We recall that, for any $1 \leq i \leq n+3$, the tautological class $\psi_{i}$ is defined as the first Chern class of the cotangent bundle, $c_{1}\left(\mathbb{L}_{i}\right)$, where $\mathbb{L}_{i}$ is the line bundle on $\overline{\mathcal{M}}_{0, n+3}$ such that over a moduli point $\left(C, x_{1}, \ldots, x_{n+3}\right)$ the fiber is the cotangent space to $C$ at $x_{i}, T_{x_{i}}^{*} C$.

In the Kapranov's model given by $\psi_{n+3}$, denote by $\mathcal{S}$ the collection of $n+2$ points in general position in $\mathbb{P}^{n}$ obtained by contraction of sections $\sigma_{i}$ of the forgetful morphism of the $n+3$ marked point. Denote by $S$ the set of indices parametrizing $\mathcal{S}$. 
Further, denote by $\mathcal{X}_{n+2,(n-2)}$ the iterated blow-up of $\mathbb{P}^{n}$ along the strict transforms of all linear subspaces $L_{I}$ of dimension at most $n-2$ spanned by sets of points $I \subset \mathcal{S}$ with $|I| \leq n-1$, ordered by increasing dimension. Notice that, in the notation of Section 1.4, for an effective divisor $D$, the iterated blown-up space $X_{n+2,(n-2)}$ along the linear subspaces that are in the base locus of $|D|$ is a resolution of singularities of $D$ (see Corollary 2.10), so it depends on the divisor we start with. However, $\mathcal{X}_{n+2,(n-2)}$ depends only on the original set of $n+2$ points in $\mathbb{P}^{n}$.

In [22] Kapranov identifies the moduli space $\overline{\mathcal{M}}_{0, n+3}$ with the projective variety $\mathcal{X}_{n+2,(n-2)}$, in the notation of Section 1 , by constructing birational maps from $\overline{\mathcal{M}}_{0, n+3}$ to $\mathbb{P}^{n}$ induced by the divisors $\psi_{i}$, for any choice of $i$ with $1 \leq i \leq n+3$.

We recall that the Picard group of $\mathcal{X}_{n+2,(n-2)}$ is spanned by a general hyperplane class and exceptional divisors, $\operatorname{Pic}\left(\mathcal{X}_{n+2,(n-2)}\right)=\left\langle H, E_{J}\right\rangle$, where $J$ is any nonempty subset of $S$ with $1 \leq|J| \leq n-1$.

Remark 4.1. In the Kapranov's model given by $\psi_{n+3}, \mathcal{X}_{n+2,(n-2)}$, there are $n+2$ Cremona transformations that are based on any subset of $n+1$ points of $S, S_{i}:=$ $S \backslash\left\{p_{i}\right\}$. The $\psi_{i}$ classes with $i \neq n+3$ correspond to the image of the Cremona transformation of a general hyperplane class $H$, based on the set $S_{i}$, denoted by $\mathrm{Cr}_{i}(H)=\mathrm{Cr}(H)$ in $(2.17)$, while $\psi_{n+3}$ corresponds to $H$.

Furthermore, we have the following identification:

$$
\Delta_{\mathcal{I}}= \begin{cases}E_{J}, & |\mathcal{I}| \leq n, \\ H_{J}, & |\mathcal{I}|=n+1 .\end{cases}
$$

where $E_{J}$ is the strict transform of the exceptional divisor obtained by blowingup the linear cycle spanned points of $J$, while $H_{J}$ is the strict transform of the hyperplane passing thought the points of $J$, namely

$$
H_{J}:=H-\sum_{\substack{I \subset J: \\ 1 \leq|I| \leq n-2}} E_{I} .
$$

The $F$-curves on $\overline{\mathcal{M}}_{0, n+3}$ correspond to partitions of the index set

$$
\mathcal{I}_{1} \sqcup \mathcal{I}_{2} \sqcup \mathcal{I}_{3} \sqcup \mathcal{I}_{4}=\{1, \ldots, n+3\} .
$$

We remark that by definition, all subsets $\mathcal{I}_{i}$ are non-empty. We denote by $F_{\mathcal{I}_{1}, \mathcal{I}_{2}, \mathcal{I}_{3}, \mathcal{I}_{4}}$ the class of the corresponding F-curve. We have the following intersection table (see [24]).

$$
F_{\mathcal{I}_{1}, \mathcal{I}_{2}, \mathcal{I}_{3}, \mathcal{I}_{4}} \cdot \Delta_{\mathcal{I}}= \begin{cases}1 & \mathcal{I}=\mathcal{I}_{i} \sqcup \mathcal{I}_{j}, \text { for some } i \neq j, \\ -1 & \mathcal{I}=\mathcal{I}_{i}, \text { for some } i \\ 0 & \text { otherwise. }\end{cases}
$$

We first describe the F-conjecture in a Kapranov's model using the coordinates of the Néron-Severi group $N^{1}\left(\mathcal{X}_{n+2,(n-2)}\right)$. Consider a general divisor on $\mathcal{X}_{n+2,(n-2)}$ of the form

$$
d H-\sum_{\substack{I \subset S: \\ 1 \leq|I| \leq n-1}} m_{I} E_{I}
$$


For a non-empty subset $I$ of the points parametrized by $S$ we define

$$
a_{I}:= \begin{cases}0 & |I| \geq n \\ 1 & |I| \leq n-1 .\end{cases}
$$

For any partition of the set of $n+3$ points $G \sqcup J \sqcup L=S$, set

$$
\begin{aligned}
A_{G, J, L}: & =d-a_{G} \cdot m_{G}-a_{J} \cdot m_{J}-a_{L} \cdot m_{L}+a_{J \sqcup L} \cdot m_{J \sqcup L} \\
& +a_{J \sqcup G} \cdot m_{J \sqcup G}+a_{L \sqcup G} \cdot m_{L \sqcup G} .
\end{aligned}
$$

Example 4.2. Consider $|G|=n$ then $J$ and $L$ consist of one element each, say $j$ and respectively $l$. Then $A_{G, J, L}$ is independent of $G$ since $A_{G, J, L}=d-m_{j}-m_{l}+m_{j l}$ for $n \geq 3$. Whenever $|G| \leq n-1$ then $A_{G, J, L}$ depends on all three subsets.

Moreover, for any two non-empty subsets of $S, I$ and $J$, set

$$
b_{I \sqcup J}:= \begin{cases}0 & |I|+|J| \geq n, \\ 1 & |I|+|J| \leq n-1 .\end{cases}
$$

For any partition $I \sqcup G \sqcup J \sqcup L=S$ set $b_{I \sqcup J \sqcup L}:=b_{I \sqcup(J \sqcup L)}$, as defined in (4.5), and

$$
\begin{aligned}
B_{I, G, J, L}: & =m_{I}-b_{I \sqcup G} \cdot m_{I \sqcup G}-b_{I \sqcup J} \cdot m_{I \sqcup J}-b_{I \sqcup L} \cdot m_{I \sqcup L}+ \\
& +b_{I \sqcup J \sqcup L} \cdot m_{I \sqcup J \sqcup L}+b_{I \sqcup G \sqcup J} \cdot m_{I \sqcup G \sqcup J}+b_{I \sqcup G \sqcup L} \cdot m_{I \sqcup G \sqcup L} .
\end{aligned}
$$

Example 4.3. If $|I|+|G|=n$ then $J$ and $L$ consist each of one element, call $j$ and $l$ respectively. In this case

- If $|I|=n-1$ then the subsets $G, J$ and $L$ consist of one element each and $B_{I, G, J, L}=m_{I}$.

- If $|I|=n-2$ then $B_{I, G, J, L}=m_{I}-m_{I \sqcup\{j\}}-m_{I \sqcup\{l\}}$.

- If $|I| \leq n-3$ then $B_{I, G, J, L}=m_{I}-m_{I \sqcup\{j\}}-m_{I \sqcup\{l\}}+m_{I \sqcup\{j\} \sqcup\{l\}}$.

Whenever $|I|+|G| \leq n-1$ then $B_{I, G, J, L}$ depends on the four subsets of the partition.

Remark 4.4. The number $A_{G, J, L}$ represents the intersection product between the divisor $D$ and the corresponding F-curve contained in a hyperplane divisor and $B_{I, G, J, L}$ represents the intersection product between the divisor $D$ and the F-curve contained in some exceptional divisor $E_{I}$.

Using the identification of boundary divisors (4.1) and the intersection table (4.2), it is easy to see that the following remark holds.

Remark 4.5 (The cone of F-nef divisors). A divisor on $\mathcal{X}_{n+2,(n-2)}$ of the form (4.3) is F-nef if the following sets of inequalities hold:

$$
\begin{aligned}
A_{G, J, L} & \geq 0, \quad \text { for any partition } G \sqcup J \sqcup L=S, \\
B_{I, G, J, L} \geq 0, & \text { for any partition } I \sqcup G \sqcup J \sqcup L=S .
\end{aligned}
$$

This cone is often referred to as the Faber cone in the literature, see e.g. [16].

Conjecture 4.6 (F-conjecture). A divisor on $\mathcal{X}_{n+2,(n-2)}$ of the form (4.3) is nef if and only if (4.6) holds.

Take a general divisor with degree and multiplicities labeled as in (4.3). We will now describe general properties of the $F$-nef divisors in a Kapranov's model that are useful in computations.

Lemma 4.7. Any F-nef divisor satisfies $d \geq m_{I} \geq 0$, for every $I \subset S$, and $m_{I} \geq m_{J}$, for every $I, J \subset S$ with $I \subset J$. 
Proof. We claim that these inequalities follow from (4.6). For $n=2$ the claim is obvious, hence we assume $n \geq 3$. In fact, the following inequalities hold:

(1) $m_{I} \geq 0$, for every non-empty set $I$ with $|I|=n-1$,

(2) $m_{I} \geq m_{J}$, for every non-empty sets $I, J$ with $I \subset J$.

Claim (1) follows from Example 4.3 and Remark 4.5. To prove claim (2) we apply induction on $|I|$. For $i \neq j$ and $i, j \notin I$ we introduce the following notations: $I_{i}:=I \sqcup\{i\}$ and $I_{i j}:=I \sqcup\{i, j\}$. For the first step of induction consider the sets $I$ and $G$ with $|I|=n-2$ and $|G|=2$. For any $i \neq j$ one has, by (4.6), that

$$
m_{I}-m_{I_{i}}-m_{I_{j}} \geq 0 \text {. }
$$

Therefore claim (2) follows from claim (1) for any $I$ with $|I|=n-2$. If $|I| \leq n-3$ the claim follows using backward induction on $|I|$. Indeed, by Example 4.3 we have

$$
m_{I}-m_{I_{i}}-m_{I_{j}}+m_{I_{i j}} \geq 0,
$$

therefore

$$
m_{I}-m_{I_{i}} \geq m_{I_{j}}-m_{I_{i j}} \geq 0 .
$$

Since $\left|I_{i}\right|=|I|+1$ and $I_{i} \subset I_{i j}$, the induction hypothesis holds for $I_{i}$, so the claim follows.

To see that $d \geq m_{I}$ we use Example 4.2 and claim (2) to obtain

$$
d \geq m_{i}+\left(m_{j}-m_{i j}\right) \geq m_{I} .
$$

4.2. The F-conjecture holds for strict transforms on $\overline{\mathcal{M}}_{0, n}$. The main result of this section is Theorem 4.8, stating that the F-conjecture holds for all divisors on $\mathcal{X}_{n+2,(n-2)}$ that are strict transforms of an effective divisor on $X_{n+2,(0)}$.

In the notation of Section $1, D$ be any effective divisor on $X_{n+2,(0)}$ and let $\tilde{D}$ denote its strict transform on $X_{n+2,(n-2)}$. A general divisor on $\mathcal{X}_{n+2,(n-2)}$, for arbitrary coefficients $d$ and $m_{I}$, is of the form (4.3) while the divisors $\tilde{D}$ have arbitrary coefficients $d$ and $m_{i}$ while $m_{I}:=k_{I}$ defined in (1.3) for any index $I$ with $|I| \geq 2$. We can consider $\tilde{D}$ a divisor on $\mathcal{X}_{n+2,(n-2)}$.

Theorem 4.8. Assume $m_{i} \geq 0$, for all $i \in S$, then Conjecture 4.6 holds for $\tilde{D}$ on $\mathcal{X}_{n+2,(n-2)}$.

Proof. To prove the claim, notice first that the effectivity of $D$ implies $\sum_{i \in S} m_{i} \leq$ $n d$ and $\sum_{i \in I} m_{i} \leq n d$, for all $I \subset S$ such that $|I|=n+1$. Moreover, since $m_{i} \geq 0$, we conclude by Theorem 2.1 and Remark 2.3.

We proved that $\tilde{D}$ is globally generated. Therefore $\tilde{D}$ is nef and in particular F-nef.

Remark 4.9. Let $f: \overline{\mathcal{M}}_{0, n} \rightarrow X_{n+2,(0)}$ be the Kapranov blow-up. For divisors of type $\tilde{D}$, the inequalities of Remark 2.3 correspond exactly to the curves generating the Mori cones of $\overline{\mathcal{M}}_{0, n}$, namely the F-curves of $\overline{\mathcal{M}}_{0, n}$ not contracted by $f$.

Corollary 4.10. For divisors of the form $\tilde{D} \geq 0$ on $\mathcal{X}_{n+2,(n-2)}$, the three properties of being F-nef, nef and globally generated are equivalent.

Remark 4.11. The divisors in (4.3) for which $m_{I}<k_{I}$ are not nef, as they intersect negatively the class of a general line on the exceptional divisor $E_{I}$, for $2 \leq|I| \leq n-1$. 
The divisors $\tilde{D}$ with $k_{I} \geq 1$, for some set $I$ with $|I| \geq 2$ are not globally generated since they contract the exceptional divisors $E_{I}$.

Remark 4.12. Studying divisors interpolating higher dimensional linear cycles, $L_{I}$ for $|I| \geq 2, m_{I}>k_{I}$, is a possible approach to the F-conjecture. Indeed, once the vanishing theorems are established by techniques developed in [5] and [12] they could be used for describing globally generated divisors or ample and nef cones of $\overline{\mathcal{M}}_{0, n}$. The description of ample divisors on $\overline{\mathcal{M}}_{g, n}$ is an important question originally asked by Mumford and conjectured by Fulton for $g=0$. In particular, [16, Conjecture 0.2] holds for $g=0$ if and only if it holds for any $g$.

\section{Part 2. Divisors obstructed by rational curves and their secants}

\section{Notation AND PRELIMINARY RESUlts}

We recall here notations and results introduced in [6].

Notation 5.1. It is classically known that there exists a unique rational normal curve $C$ of degree $n$ passing through $n+3$ general points of $\mathbb{P}^{n}$ [31]. Let $\sigma_{t}=\sigma_{t}(C)$ denote the $t$-th secant variety of $C$, namely the Zariski closure of the union of $t$ secant $(t-1)$-planes. In this notation we have $\sigma_{1}=C$. For every $I \subset\{1, \ldots, n+3\}$ with $|I|=r+1,-1 \leq r \leq n$, let

$$
\mathrm{J}\left(L_{I}, \sigma_{t}\right)
$$

be the join of the linear cycle $L_{I}$ and $\sigma_{t}$; this is a cone with vertex $L_{I}$. We use the conventions $|\emptyset|=0$ and $\sigma_{0}=\emptyset$.

The dimension of the variety $\mathrm{J}\left(L_{I}, \sigma_{t}\right)$ is

$$
r=r_{I, \sigma_{t}}:=\operatorname{dim} \mathrm{J}\left(L_{I}, \sigma_{t}\right)=|I|+2 t-1 .
$$

Notice if $t=0$, then the join $\mathrm{J}\left(L_{I}, \sigma_{0}\right)$ is the linear cycle spanned by the points parametrized by $I, L_{I}$, while if $I=\phi$, then the join $\mathrm{J}\left(L_{I}, \sigma_{t}\right)=\sigma_{t}$ is the secant variety $\sigma_{t}$.

\subsection{Strong base locus lemma for divisors on $X_{s+3,(0)}$.}

Lemma 5.2 ([6, Lemma 4.1]). For any effective divisor $D$ as in (1.2) and for $r_{I, \sigma_{t}} \leq n-1$, the multiplicity of containment in $\mathrm{Bs}(|D|)$ of the strict transform in $X_{s,(0)}$ of the subvariety $\mathrm{J}\left(L_{I}, \sigma_{t}\right)$ is the integer

$$
k_{I, \sigma_{t}}=k_{I, \sigma_{t}}(D):=\max \left\{0, t \sum_{i=1}^{n+3} m_{i}+\sum_{i \in I} m_{i}-((n+1) t+|I|-1) d\right\} .
$$

In particular, the formula (5.2) for a linear cycle $L_{I}$ reads

$$
k_{I}=\sum_{i \in I} m_{i}-(|I|-1) d
$$

while, for the secant variety $\sigma_{t}$, it reads

$$
k_{\sigma_{t}}=t \sum_{i=1}^{n+3} m_{i}-((n+1) t-1) d .
$$

We will now prove a stronger result that will play a crucial role in Section 3, particularly in Proposition 6.15. 
OLIVIA DUMITRESCU AND ELISA POSTINGHEL

Proposition 5.3. Let $D$ be an effective divisor on $X_{s,(0)}$. Let $p$ be a point on the variety $\mathrm{J}\left(L_{I}, \sigma_{t}\right)$ that does not lie on any smaller join $\mathrm{J}\left(L_{I^{\prime}}, \sigma_{t^{\prime}}\right) \subsetneq \mathrm{J}\left(L_{I}, \sigma_{t}\right)$, $I^{\prime} \subset I, t^{\prime} \leq t$. Then $p$ is contained in the base locus of the general member of the linear system $|D|$ with multiplicity precisely equal to $k_{I, \sigma_{t}}$.

Proof. We first prove the claim for $t=0$ when the join $\mathrm{J}\left(L_{I}, \sigma_{0}\right)$ is the linear cycle $L_{I}$ that is in the base locus of the general member of $|D|$ with multiplicity of containment equal to $k_{I}=k_{I}(D)$, see [12, Proposition 4.2].

Let us assume, by contradiction, that there is a point $p$ of $L_{I}$ with multiplicity of containment at least $k_{I}+1$. Let $I(r)$ be the largest index set such that $p \in L_{I(r)}$. Lemma 4.2 of [7] implies $r \geq 1$. We introduce the following notation:

$$
K_{I(r)}=K_{I(r)}(D):=\sum_{i \in I(r)} m_{i}-r d .
$$

Consider first the case when $K_{I(r)} \geq 1$ and note that $k_{I(r)}=K_{I(r)}$. For $r \geq 2$, let $I(r-2)$ be a subset of $I(r)$, of cardinality equal to $r-1$, and take $H_{I(r-2)}$ a general hyperplane passing through $p$ and all points of $I(r-2)$. For $r=1, H_{I(-1)}$ denotes the hyperplane passing through the point $p$. For every $r \geq 1$, consider the divisor $D^{\prime}$, defined as follows, and denote by $d^{\prime}$ and $m_{i}^{\prime}$ its corresponding degree and multiplicities:

$$
D^{\prime}:=D+K_{I(r)} H_{I(r-2)} .
$$

By assumption, the general member of $\left|D^{\prime}\right|$ contains the point $p$ with multiplicity at least 1 . We can compute the multiplicity of containment of $L_{I(r)}$ in the base locus of $D^{\prime}$ :

$$
\begin{aligned}
K_{I(r)}\left(D^{\prime}\right) & =\sum_{i \in I(r)} m_{i}^{\prime}-r d^{\prime} \\
& =\sum_{i \in I(r)} m_{i}-r d+(r-1) K_{I(r)}-r K_{I(r)} \\
& =K_{I(r)}-K_{I(r)} \\
& =0
\end{aligned}
$$

Consider now the case $K_{I(r)}<0$. Let $H_{I(r)}$ denote a hyperplane containing all points of $I(r)$. Define the divisor

$$
D^{\prime \prime}:=D-K_{I(r)} H_{I(r)} .
$$

A similar computation shows the following

$$
\begin{aligned}
K_{I(r)}\left(D^{\prime \prime}\right) & =\sum_{i \in I(r)} m_{i}^{\prime \prime}-r d^{\prime \prime} \\
& =\sum_{i \in I(r)} m_{i}-r d-(r+1) K_{I(r)}+r K_{I(r)} \\
& =K_{I(r)}-K_{I(r)} \\
& =0 .
\end{aligned}
$$

In the above cases we reduced to the case when $D$ is a divisor with $k_{I(r)}=0$ whose general member has a base point, $p$. We now prove by induction on $r$ that this leads to a contradiction. 
We discuss separately the case $r=1$ as the first induction step. The line $L_{I(1)}$ is not contained in the base locus of $D$ by Lemma 5.2 for $t=0$ and $r=1$. However, the intersection multiplicity between the line $L_{I(1)}$ and $D$ is negative, a contradiction.

In general, we assume that the statement holds for linear cycles of dimension $r-1$ and we prove that it holds for linear cycles of dimension $r$. The point $p$ can not lie on any smaller linear cycle contained in some $L_{I(r-1)} \subset L_{I(r)}$, by the induction assumption. Therefore, $p$ is a point inside the interior of the cycle $L_{I(r)}$, namely $p \in L_{I(r)} \backslash \bigcup_{I(r-1) \subset I(r)} L_{I(r-1)}$. It is easy to see that whenever $k_{I(r)} \geq 0$, then $k_{J} \geq 0$ for any subset $J$. This implies that for any subset $I(r-1) \subset I(r)$ of cardinality $r$, the divisor $D$ contains the linear cycle $L_{I(r-1)}$ in its base locus with multiplicity $k_{I(r-1)} \geq 0$. We consider $l$ a general line in $L_{I(r)}$ passing trough $p$. We observe that the multiplicity of intersection between $l$ and the divisor $D$ is at most

$$
\begin{aligned}
d-\sum_{I(r-1) \subset I(r)} k_{I(r-1)}-1 & =d-\sum_{I(r-1) \subset I(r)}\left(\sum_{i \in I(r-1)} m_{i}-(r-1) d\right)-1 \\
& =r\left(\sum_{i \in I(r)} m_{i}-r d\right)-1 \\
& =-1 .
\end{aligned}
$$

Since the family of lines passing through the point $p$ covers the linear cycle $L_{I(r)}$ one obtains that $L_{I(r)}$ is in the base locus of the divisor $D$ that is a contradiction with Lemma 5.2, since $k_{I(r)}=0$ (for $t=0$ ).

If $t \geq 1$, the proof follows by an argument similar to the one used in [12, Proposition 4.2 .

5.2. The blown-up space $X_{n+3,(n-2)}^{\sigma}$. In this section we extend Question 1.10 to the case of effective divisors on the blow-up of $\mathbb{P}^{n}$ at $n+3$ general points. Take

$$
D=d H-\sum_{i=1}^{n+3} m_{i} E_{i} \geq 0,
$$

a divisor on $X_{n+3,(0)}$, the blown-up $\mathbb{P}^{n}$ at $n+3$ base points, with $d, m_{i} \geq 0$.

We will obtain $X_{n+3,(n-2)}^{\sigma}$ from $X_{n+3,(0)}$ by iterated blow-up along the subvarieties that are contained in the base locus of $D$, that are the strict transforms of $\mathrm{J}\left(L_{I}, \sigma_{t}\right)$, see Lemma 5.2. The pairwise intersections of such subvarieties with some constraints on the index sets parametrizing the vertices, is computed in [2, Proposition 5.6]. For the sake of completeness we state below this result in our notation.

Proposition 5.4 ([2, Proposition 5.6]). Let $I_{1}, I_{2} \subset\{1, \ldots, n+3\}$ be index sets such that $I_{1} \cap I_{2}=\emptyset$. Let $t_{1}, t_{2} \geq 0$ be integers such that

$$
\begin{aligned}
& r_{I_{1}, \sigma_{t_{1}}}=r_{I_{2}, \sigma_{t_{2}}}, \\
& r_{I_{i}, \sigma_{t_{i}}} \leq n-1, \forall i \in\{1,2\}, \\
& 2 r_{I_{i}, \sigma_{t_{i}}} \leq 2 n-\left(\left|I_{1}\right|+\left|I_{2}\right|\right), \forall i \in\{1,2\} .
\end{aligned}
$$

Then

$$
\mathrm{J}\left(L_{I_{1}}, \sigma_{t_{1}}\right) \cap \mathrm{J}\left(L_{I_{2}}, \sigma_{t_{2}}\right)=\bigcup_{J} \mathrm{~J}\left(L_{J}, \sigma_{t_{J}}\right),
$$


where the union is taken over all subsets $J \subseteq I_{1} \cup I_{2}$ satisfying

$$
2\left|\left(I_{i} \cup J\right) \backslash\left(I_{i} \cap J\right)\right|=\left|I_{1}\right|+\left|I_{2}\right|, \forall i \in\{1,2\},
$$

and for every such $J, t_{J}$ is the integer defined by the following equation

$$
2 r_{J, \sigma_{t_{J}}}=2 r_{I_{i}, \sigma_{t_{i}}}-\left(\left|I_{1}\right|+\left|I_{2}\right|\right) \text {. }
$$

Notation 5.5. For the sake of simplicity let us denote $Y^{\sigma}:=X_{n+3,(n-2)}^{\sigma}$ and $X:=X_{n+3,(0)}$. For any effective divisor $D$ of the form (5.5), let

$$
\pi^{\sigma}: Y^{\sigma} \rightarrow X
$$

be the iterated blow-up of $X:=X_{n+3,(0)}$ along (the strict transforms of) all varieties $\mathrm{J}\left(L_{I}, \sigma_{t}\right), t \geq 0,|I| \geq 0$, such that $r_{I, \sigma_{t}} \leq n-2$ and $k_{I, \sigma_{t}}>0$ in increasing dimension, composed with a contraction of the strict transforms of the divisors $\mathrm{J}\left(L_{I}, \sigma_{t}\right)$ with $r_{I, \sigma_{t}}=n-1$ and $k_{I, \sigma_{t}}>0$.

The latter divisors were described in [6, Section 3.2]. The space $X_{n+3,(n-2)}^{\sigma}$ is constructed by Araujo and Massarenti in their recent article [2, Section 5] in order to give explicit $\log$ Fano structures on $X_{n+3,(0)}$ (see [2, Propositions 5.8, 5.11]).

Notation 5.6. We denote by $E_{I, \sigma_{t}}$ the exceptional divisors, for all $t \geq 0,|I| \geq 0$, $r_{I, \sigma_{t}} \leq n-1$.

It is immediate to see, using [6, Lemma 4.1], that the strict transform on $Y^{\sigma}$ of $D$ is given by

$$
\tilde{D}^{\sigma}:=d H-\sum_{i} m_{i} E_{i}-\sum_{r=1}^{n-1} \sum_{\substack{I, t: \\ r_{I, \sigma_{t}}=r}} k_{I, \sigma_{t}} E_{I, \sigma_{t}} .
$$

We stress the fact that the space $Y^{\sigma}$ depends on the divisor $D$.

5.3. Conjectures on vanishing cohomology for divisors on $X_{n+3,(n-2)}^{\sigma}$. Let $D$ be an effective divisor on $\mathbb{P}^{n}$ blown-up in $n+3$ general points.

Question 5.7. Consider the divisor $\tilde{D}_{\sigma}$ defined in (5.7) as the strict transform of $D$ on $X_{n+3,(n-2)}^{\sigma}$. Does $h^{i}\left(\tilde{D}^{\sigma}\right)$ vanish for all $i \geq 1$ ?

5.3.1. Related questions. Another challenge would be to compute the Euler characteristic of $\tilde{D}^{\sigma}$. In what follows we would like to propose a candidate for such a number, namely the so called secant linear virtual dimension for linear systems of hypersurfaces of $\mathbb{P}^{n}$ interpolating $n+3$ general points with assigned multiplicity, or equivalently of linear systems $|D|$. This number was introduced in [6].

Definition 5.8 ([6, Definition 6.1]). Let $D$ be a divisor on $X_{n+3,(0)}$ as in (5.5). The secant linear virtual dimension of $|D|$ is the number

$$
\sigma \operatorname{ldim}(D):=\sum_{I, \sigma_{t}}(-1)^{|I|}\left(\begin{array}{c}
n+k_{I, \sigma_{t}}-r_{I, \sigma_{t}}-1 \\
n
\end{array}\right),
$$

where the sum ranges over all indexes $I \subset\{1, \ldots, n+3\}$ and $t$ such that $0 \leq t \leq l+\epsilon$, $n=2 l+\epsilon$ and $0 \leq|I| \leq n-2 t$. The integers $k_{I, \sigma_{t}}$ and $r_{I, \sigma_{t}}$ are defined in (5.2) and in (5.1) respectively.

Conjecture 5.9. The Euler characteristic of the divisor $\tilde{D}^{\sigma}$, defined in (5.7) as the strict transform of $D$ in $X_{n+3,(n-2)}^{\sigma}$, is

$$
\chi\left(\tilde{D}^{\sigma}\right)=\sigma \operatorname{ldim}(D) .
$$


The above questions are related to the dimensionality problem for linear systems of divisors of the form (1.2) and in particular to the Fröberg-Iarrobino conjectures $[15,21]$, which give a predicted value for the Hilbert series of an ideal generated by $s$ general powers of linear forms in the polynomial ring with $n+1$ variables. We refer to [6, Section 2.1] for a more detailed account on this. In [6] the following conjectural answer to this problem was given in terms of Definition 5.8.

Conjecture 5.10 ([6, Conjecture 6.4]). Set $X=X_{n+3,(0)}$ and let $D$ be as in (5.5). Then

$$
h^{0}\left(X, \mathcal{O}_{X}(D)\right)=\max \{0, \sigma \operatorname{ldim}(D)\} .
$$

Previous work $[5,12]$ contains a proof that Question 5.7 admits an affirmative answer, as well as proofs of Conjectures 5.9 and 5.10, for divisors satisfying the bound (1.5), namely those that do not contain positive multiples of the rational normal curve of degree $n$ in the base locus, nor joins $\mathrm{J}\left(I, \sigma_{t}\right)$. The approach adopted was based on the study of the normal bundles of the exceptional divisors of linear cycles, $L_{I}$, and vanishing cohomologies of strict transforms on $X_{s,(n-2)}$, the blow-up along the linear cycles, see Theorem 1.9.

We believe that a proof of the above conjectures for the general case for $s=n+3$ would rely on the study of the normal bundles to the joins $\mathrm{J}\left(I, \sigma_{t}\right)$. We plan to develop this approach in future work.

On a more general note, we would like to point out that the construction of $X_{s,(n-2)}^{\sigma}$ and Question 5.7 and Conjectures 5.10 and 5.9 could be generalised to $\mathbb{P}^{n}$ blown-up in arbitrary number of points in linearly general position.

\section{Explicit PROOF OF LOG ABUNDANCE FOR EFFECTIVE DIVISORS ON $X_{n+3,(0)}$}

In this section we give an explicit constructive proof of the log abundance conjecture for pairs $\left(X_{n+3,(0)}, D\right)$ where $D$ is any effective divisor. We recall that effective divisors on $X_{s,(0)}$, with $s \leq n+3$ were classified in [6]. The case where $D$ has only linear base locus is covered in Theorem 3.3. In this section we complete the picture by studying divisors $D$ that contains also the rational normal curve of degree $n$ through the $n+3$ points, as well as possibly joins between linear cycles and its secants (see Notation 5.1).

Theorem 6.1. Let $n>3$ be the dimension of the space and $\epsilon \in \mathbb{Q}$, with $0 \leq \epsilon \ll 1$. Let $D=d H-\sum_{i=1}^{s} m_{i} E_{i}$ be a general effective divisor on $X=X_{s,(0)}$ and assume that (3.2) holds. Then the pair $(X, \Delta)$ is lc.

Set $X:=X_{n+3,(0)}$ and let $D$ on $X$ be a divisor of the form

$$
D=d H-\sum_{i=1}^{n+3} m_{i} E_{i} \geq 0 .
$$

Write $\Delta=\epsilon D$, for $0 \leq \epsilon \ll 1$ and assume it satisfies condition (3.2) of Theorem 3.3 .

Remark 6.2. Note that, (3.2) implies that $D$ is big (in fact it lies in the interior of the effective cone, see [6, Theorem 5.1]). In fact we can use (6.4) that gives

$$
\epsilon k_{I(n-2 t), \sigma_{t}} \leq-n-1<0,
$$

for all $I(n-2 t), t \geq 1$. Moreover one can similarly check that

$$
\epsilon k_{I(n-2 t-1), \sigma_{t}} \leq 0
$$


for all $I(n-2 t-1), t \geq 1$, hence $D$ is movable, see [6, Theorem 5.3]. In particular the general element of $|D|$ is irreducible.

Remark 6.3. The argument developed in Section 3.1 applies also to the case $s=n+3$ and $k_{C}:=k_{C}(D)=0$ (see (5.2) for the definition of this number), namely to divisors $D$ on $X_{n+3,(0)}$ that have only linear base locus and for which (2.2) is satisfied. Indeed under this condition and (3.2), $D$ is movable (see [6, Theorem 5.3]) and its strict transform on $X_{n+3,(n-2)}$, the iterated blow-up along the linear cycles, is globally generated by Theorem 2.1. Moreover the lc condition on the pair $(X, \epsilon \Delta)$ can be verified in the same manner as in the case with (2.2), as in Section 3.1.

From now on we will assume that $k_{C}(D) \geq 1$.

6.1. Constructing a log resolution of $(X, \epsilon D)$. The following result computes the pairwise intersections of the joins $\mathrm{J}\left(L_{I}, \sigma_{t}\right)$.

Proposition 6.4. Let $\mathrm{J}\left(L_{I_{1}}, \sigma_{t_{1}}\right)$ and $\mathrm{J}\left(L_{I_{2}}, \sigma_{t_{2}}\right)$ be any join varieties of the same dimension $r_{I_{1}, \sigma_{t_{1}}}=r_{I_{2}, \sigma_{t_{2}}}$. If $2 r_{I_{1}, \sigma_{t_{1}}} \leq n-1$,

then

$$
\mathrm{J}\left(L_{I_{1}}, \sigma_{t_{1}}\right) \cap \mathrm{J}\left(L_{I_{2}}, \sigma_{t_{2}}\right)=\bigcup_{J} \mathrm{~J}\left(L_{J}, \sigma_{t_{J}}\right),
$$

for some subsets $J \subseteq I_{1} \cup I_{2}$.

Proof. Assume first of all that $I_{1} \cap I_{2}=\emptyset$. We have

$$
0 \leq 2(n-1)-2\left(r_{I_{1}, \sigma_{t_{1}}}+r_{I_{2}, \sigma_{t_{2}}}\right),
$$

which gives

$$
2 r_{I_{i}, \sigma_{t_{i}}} \leq 2 n-\left(r_{I_{1}, \sigma_{t_{1}}}+1\right)-\left(r_{I_{2}, \sigma_{t_{2}}}+1\right), \forall i \in\{1,2\} .
$$

Therefore, since $\left|I_{i}\right| \leq r_{I_{i}, \sigma_{t_{i}}}+1$, we have $2 r_{I_{i}, \sigma_{t_{i}}} \leq 2 n-\left(\left|I_{1}\right|+\left|I_{2}\right|\right), i=1,2$, hence we are in the hypotheses of Proposition 5.4. This concludes the proof of the statement in the case when $I_{1}, I_{2}$ are disjoint. Indeed at each step of blow-up, the intersection of any two subvarieties that are being blown-up is a union of smaller subvarieties that have been previously blown-up.

Assume now that $I_{1} \cap I_{2}=: I_{12} \neq \emptyset$. Set $I_{i}^{\prime}:=I_{i} \backslash I_{12}$, for $i=1,2$. Notice that

$$
r_{I_{i}^{\prime}, \sigma_{t_{i}}}=r_{I_{i}, \sigma_{t_{i}}}-\left|I_{12}\right|, \forall i \in\{1,2\} .
$$

Moreover,

$$
\mathrm{J}\left(\mathrm{J}\left(L_{I_{i}^{\prime}}, \sigma_{t_{i}}\right), L_{I_{12}}\right)=\mathrm{J}\left(L_{I_{i}}, \sigma_{t_{i}}\right), \forall i \in\{1,2\}
$$

Also,

$$
\mathrm{J}\left(\mathrm{J}\left(L_{I_{1}^{\prime}}, \sigma_{t_{1}}\right), L_{I_{12}}\right) \cap \mathrm{J}\left(\mathrm{J}\left(L_{I_{2}^{\prime}}, \sigma_{t_{2}}\right), L_{I_{12}}\right)=\mathrm{J}\left(\mathrm{J}\left(L_{I_{1}^{\prime}}, \sigma_{t_{1}}\right) \cap \mathrm{J}\left(L_{I_{2}^{\prime}}, \sigma_{t_{2}}\right), L_{I_{12}}\right) .
$$

One proves the two last equalities of subvarieties using induction on $\left|I_{12}\right|$ based on the case $\left|I_{12}\right|=0$ for which the statement is obvious. With a similar argument as the one employed in the first case, we obtain that

$$
\begin{aligned}
2 r_{I_{i}^{\prime}, \sigma_{t_{i}}} & \leq 2 n-\left(r_{I_{1}^{\prime}, \sigma_{t_{1}}}+1\right)-\left(r_{I_{2}^{\prime}, \sigma_{t_{2}}}+1\right)-4\left|I_{12}\right|, \\
& \left.\leq 2 n-\left(\left|I_{1}^{\prime}\right|+\left|I_{2}^{\prime}\right|\right)\right),
\end{aligned}
$$


for $i=1,2$. Therefore,

$$
\mathrm{J}\left(L_{I_{1}}, \sigma_{t_{1}}\right) \cap \mathrm{J}\left(L_{I_{2}}, \sigma_{t_{2}}\right)=\mathrm{J}\left(\bigcup_{J} \mathrm{~J}\left(L_{J}, \sigma_{t_{J}}\right), L_{I_{12}}\right)=\bigcup_{J} \mathrm{~J}\left(L_{J \cup I_{12}}, \sigma_{t_{J}}\right),
$$

where the union is taken over all subsets $J \subseteq I_{1}^{\prime} \cup I_{2}^{\prime}$ satisfying $2\left|\left(I_{i}^{\prime} \cup J\right) \backslash\left(I_{i} \cap J\right)\right|=$ $\left|I_{1}^{\prime}\right|+\left|I_{2}^{\prime}\right|, \forall i \in\{1,2\}$, and for every such $J, t_{J}$ is the integer defined by the following equation $2 r_{J, \sigma_{t_{J}}}=2 r_{I_{i}^{\prime}, \sigma_{t_{i}}}-\left(\left|I_{1}^{\prime}\right|+\left|I_{2}^{\prime}\right|\right)$. The first equality of (6.2) follows from Proposition 5.4, the second inequality is an application of (6.1).

Notation 6.5. For every $n$, let $\mathcal{T}:=\left\{(I, t): 1 \leq r_{I, \sigma_{t}} \leq n-2\right\}$ be the set parametrizing all subvarieties $\mathrm{J}\left(L_{I}, \sigma_{t}\right)$ of $\mathbb{P}^{n}$ of dimension between 1 and $n-2$.

(1) If $n$ is even, say $n=2 \nu$, with $\nu \geq 2$, we consider the following set

$$
\mathcal{C}^{\text {even }}:=\left\{(I, t) \in \mathcal{T}: 2 r_{I, \sigma_{t}} \leq 2 \nu-1 \text { if } I \neq \emptyset\right\} .
$$

In other terms, $(I, t) \in \mathcal{C}^{\text {even }}$ if $I=\emptyset$ and $1 \leq t<\nu$ or if $I \neq \emptyset$ and $|I|+2 t \leq \nu$.

(2) If $n$ is odd, say $n=2 \nu+1$, with $\nu \geq 2$, we consider the following set

$$
\mathcal{C}^{\text {odd }}:=\left\{(I, t) \in \mathcal{T}: 2 r_{I, \sigma_{t}} \leq 2 \nu \text { if } I \neq \emptyset,\{1\}\right\} .
$$

In other terms, $(I, t) \in \mathcal{C}^{\text {even }}$ if $I=\emptyset,\{1\}$ and $1 \leq t<\nu$ or if $I \neq \emptyset,\{1\}$ and $|I|+2 t \leq \nu+1$.

We consider

$$
\pi^{\sigma}: Y^{\sigma} \rightarrow X
$$

the iterated blow-up $Y^{\sigma}:=X_{n+3,(n-2)}^{\sigma}$ of $X:=X_{2 \nu+3,(0)}$ (cf. Notation 5.5) along all subvarieties $\mathrm{J}\left(L_{I}, \sigma_{t}\right)$ such that $(I, t) \in \mathcal{C}^{\text {even }}$ if $n$ is even, and such that $(I, t) \in$ $\mathcal{C}^{\text {odd }}$ if $n$ is odd, in increasing dimension. We denote by $E_{I, \sigma_{t}}$ the corresponding exceptional divisors.

Let us denote $\operatorname{Exc}\left(\pi^{\sigma}\right)=\sum_{(I, t) \in \mathcal{C}^{\text {even }}} E_{I, \sigma_{t}}$ if $n$ is even and $\operatorname{Exc}\left(\pi^{\sigma}\right)=$ $\sum_{(I, t) \in \mathcal{C}^{\text {odd }}} E_{I, \sigma_{t}}$ if $n$ is odd.

Proposition 6.6. For any $n$, the blown-up space $Y^{\sigma}$ is smooth. Moreover $\operatorname{Exc}\left(\pi^{\sigma}\right)$ is simple normal crossing.

Proof. At each level of the blow-up, the center is a disjoint union of smooth subvarieties. Indeed, if $\mathrm{J}\left(L_{I_{1}}, \sigma_{t_{1}}\right)$ and $\mathrm{J}\left(L_{I_{2}}, \sigma_{t_{2}}\right)$ are two subvarieties such that $2 r_{I_{1}, \sigma_{t_{1}}}=2 r_{I_{2}, \sigma_{t_{2}}} \leq n-1$, then we conclude by Proposition 6.4. Otherwise, if $2 r_{I, \sigma_{t}} \geq n$, the only subvarieties that we blow-up are the strict transforms of the secant varieties $\sigma_{t}$ 's, with $\frac{\nu+1}{2} \leq t \leq \nu$, in the case $n=2 \nu$, and the strict transforms of the $\sigma_{t}$ 's and of the pointed cones $\mathrm{J}\left(L_{\{1\}}, \sigma_{t}\right)$, with $\frac{\nu}{2}+1 \leq t \leq \nu$, if $n=2 \nu+1$. It is easy to see that such pointed cones intersect along smaller joins of the same form, that have been previously blown-up.

6.1.1. Properties of the strict transform of $|\epsilon D|$. Denote by $\tilde{D}^{\sigma}$ the strict transform of $D$, as in (5.7):

$$
\tilde{D}^{\sigma}:=d H-\sum_{i} m_{i} E_{i}-\sum_{r=1}^{n-2} \sum_{\substack{I, t: \\ r_{I}, \sigma_{t}=r}} k_{I, \sigma_{t}} E_{I, \sigma_{t}} .
$$


Remark 6.7. Notice that, as in the only linearly obstructed case (handled in Section 3.1), the integer $r$ in the above summation of exceptional divisors ranges up to $n-2$, as $D$ is movable (cfr. [6, Theorem 5.3]).

We use the sets $\mathcal{C}^{\text {even }}$ and $\mathcal{C}^{\text {odd }}$ introduced in Notation 6.5 in the following lemma.

Lemma 6.8. For any $n, s=n+3$ and $D$ as in Theorem 3.3, if $k_{I, \sigma_{t}}>0$, then $2 r_{I, \sigma_{t}}<n-1$. In particular such a pair $(I, t) \in \mathcal{T}$ belongs to $\mathcal{C}^{\text {even }}$ if $n$ is even, and to $\mathcal{C}^{\text {odd }}$, if $n$ is odd.

Proof. Let us compute

$$
\begin{aligned}
\epsilon k_{C}= & \epsilon \max \left\{0,\left(\sum_{i=1}^{n-3} m_{i}-(n-3) d\right)+\left(m_{n-2}+m_{n-1}-d\right)+\right. \\
& \left.+\left(m_{n}+m_{n+1}-d\right)+\left(m_{n+2}+m_{n+3}-d\right)\right\} \\
\leq & -2(n-3)+3(n-2) \\
= & n-3,
\end{aligned}
$$

where $k_{C}:=k_{\sigma_{1}}$ is the multiplicity of containment of the rational normal curve of degree $n$. If $k_{C}>0$, we also compute

$$
\begin{aligned}
\epsilon\left(k_{C}-d\right) & =\epsilon\left(\sum_{i=1}^{n-1} m_{i}-(n-1) d+\left(m_{n}+m_{n+1}-d\right)+\left(m_{n+2}+m_{n+3}-d\right)\right) \\
& \leq-2(n-1)+2(n-2) \\
& =-4 .
\end{aligned}
$$

Both of the above inequalities follow from (3.2), second line, and the inequality (3.3), computed in the proof of Theorem 3.3. Hence, for every $t \geq 0$, we obtain

$$
\begin{aligned}
\epsilon k_{\sigma_{t}} & =\epsilon \max \left\{0, k_{C}+(t-1)\left(k_{C}-d\right)\right\} \\
& \leq \max \{0, n-3-4(t-1)\} .
\end{aligned}
$$

Furthermore, for every $I$ with $|I| \geq 0$ and $t \geq 0$, we obtain

$$
\begin{aligned}
\epsilon k_{I, \sigma_{t}} & =\epsilon \max \left\{0, k_{\sigma_{t}}+\sum_{i \in I} m_{i}-(|I|) d\right\} \\
& \leq \max \{0,(n-3-4(t-1))-2|I|\} \\
& =\max \{0,(n-|I|-2 t+1)-(|I|+2 t)\} .
\end{aligned}
$$

Hence, for every variety $\mathrm{J}\left(L_{I}, \sigma_{t}\right) \subset \mathbb{P}^{n}$ with $r_{I, \sigma_{t}}=|I|+2 t-1 \leq n$, we obtain

$$
\epsilon k_{I, \sigma_{t}} \leq \max \left\{0, n-1-2 r_{I, \sigma_{t}}\right\} .
$$

Corollary 6.9. In the same notation as Lemma 6.8, the divisor $\tilde{D}^{\sigma}$ intersects transversally each exceptional divisors.

Theorem 6.10. For any $n, s=n+3$ and $D$ as in Theorem 3.3. If the divisor $D$ is general, then $\tilde{D}^{\sigma}$ is base point free.

We will show Theorem 6.10 in Section 6.2 (if $n$ is even) and in Section 6.3 (is $n$ is odd). The next corollary follows from Bertini's Theorem. 
Corollary 6.11. In the notation of Theorem 6.10, the general element of $\left|\tilde{D}^{\sigma}\right|$ is smooth.

6.2. Proof of Theorem 6.10, case $n$ even. Write $n=2 \nu, \nu \geq 2$. Recall that the strict transform on $X:=X_{2 \nu+3,(0)}$ of the secant variety $\sigma_{\nu} \subset \mathbb{P}^{2 \nu}$ is the fixed divisor

$$
\Sigma:=(\nu+1) H-\nu \sum_{i=1}^{2 \nu+3} E_{i} .
$$

Proposition 6.12. In the above notation, the strict transform $\tilde{\Sigma}^{\sigma}$ of $\Sigma$ on $Y^{\sigma}$ is smooth.

Proof. Recall that the singular locus of $\sigma_{\nu} \subset \mathbb{P}^{2 \nu}$ is $\sigma_{\nu-1} \subset \sigma_{\nu}$ and, more precisely, the non-reduced union of all joins $\mathrm{J}\left(L_{I}, \sigma_{t}\right)$ such that $k_{I, \sigma_{t}}(\Sigma)>1$. In particular we compute the following multiplicities. For every $I$ and $t$ such that $|I|, t \geq 0$, we have

$$
k_{I, \sigma_{t}}(\Sigma)=\max \{0, \nu-|I|-t+1\} .
$$

All subvarieties $\mathrm{J}\left(L_{I}, \sigma_{t}\right)$ such that $|I|+t \leq \nu$ have been blown-up, hence we conclude that $\pi^{\sigma}$ is a resolution of the singularities of $\Sigma$.

Choose $\alpha \in \mathbb{N}$ such that

$$
\frac{k_{C}(D)}{\nu} \leq \alpha \leq \min _{1 \leq i \leq 2 \nu+3}\left\{\frac{m_{i}}{\nu}, d-m_{i}\right\} .
$$

Lemma 6.13. Under the assumptions of Theorem 3.3, such an integer $\alpha$ exists.

Proof. It is enough to prove that $\frac{k_{C}(D)}{\nu} \leq \frac{m_{i}}{\nu}-1$ and that $\frac{k_{C}(D)}{\nu} \leq d-m_{i}-1$ for all $i \in\{1 \ldots, 2 \nu+3\}$. The first statement follows from the following computation:

$$
\epsilon k_{C}(D) \leq 2 \nu-3 \leq 2 \nu-1-\epsilon \nu \leq \epsilon\left(m_{i}-\nu\right) .
$$

The first inequality follows from (6.4). The second inequality follows from the assumption $\epsilon \ll 1$; in fact it is enough to take $\epsilon \leq \frac{4}{n}=\frac{2}{\nu}$. The last inequality follows from (3.2). Similarly, one proves the second statement by observing that

$$
\epsilon k_{C}(D) \leq 2 \nu-3 \leq 2 \nu-\epsilon \nu \leq \epsilon \nu\left(d-m_{i}-1\right) .
$$

The last inequality follows from (3.3).

Consider the linear system

$$
\left|D^{\prime}\right|:=|D-\alpha \Sigma|=\left|d^{\prime} H-\sum_{i=1}^{2 \nu+3} m_{i}^{\prime} E_{i}\right|,
$$

with $d^{\prime}:=d-\alpha(\nu+1)$ and $m_{i}^{\prime}:=m_{i}-\alpha \nu$, for all $i \in\{1 \ldots, 2 \nu+3\}$. We have

$$
\left|D^{\prime}\right|+\alpha \Sigma \subseteq|D| .
$$

Proposition 6.14. In the above notation, the linear system $\left|D^{\prime}\right|$ is non-empty. Moreover $\left|D^{\prime}\right|$ has only linear base locus and the strict transform in $Y^{\sigma}$ of $\left|D^{\prime}\right|$ is base point free. 
Proof. For every $i \in\{1 \ldots, 2 \nu+3\}$, since by (6.6) we have $\alpha \leq \frac{m_{i}}{\nu}$ and $\alpha \leq d-m_{i}$, then $m_{i}^{\prime} \geq 0$ and $d \geq m_{i}^{\prime}$ respectively. Moreover, let us compute

$$
\begin{aligned}
k_{C}\left(D^{\prime}\right) & =\max \left\{0, \sum_{i=1}^{2 \nu+3}\left(m_{i}-\alpha \nu\right)-2 \nu(d-\alpha(\nu+1))\right\} \\
& =\max \left\{0, k_{C}(D)-\alpha \nu\right\} \\
& =0 .
\end{aligned}
$$

The last equality follows from (6.6): $\alpha \geq \frac{k_{C}(D)}{\nu}$. This proves the first statement, namely that $\Delta^{\prime}$ is effective, see [6, Theorem 5.1].

To prove the second statement, notice that if $|I| \leq \nu$, then the pair $(I, 0) \in \mathcal{C}^{\text {even }}$, hence the corresponding linear subspace $L_{I} \subset \mathbb{P}^{2 \nu}$ has been blown-up. Otherwise, if $|I| \geq \nu+1$, we claim that $k_{I}\left(D^{\prime}\right)=0$. Theorem 2.1 implies the second statement, namely that $\tilde{D}^{\sigma}$ is a globally generated divisor. To prove the claim, for every $I$ we choose $J \subset I$ with $|J|=\nu$ and we compute

$$
\begin{aligned}
\epsilon k_{I}(D) & =\max \left\{0, \epsilon\left(\sum_{i \in I} m_{i}-(|I|-1) d\right)+\epsilon \alpha(|I|-\nu-1)\right\} \\
& =\max \left\{0, \epsilon\left(\sum_{i \in J} m_{i}-(\nu-1) d\right)+\epsilon\left(\sum_{i \in I \backslash J}\left(m_{i}-d\right)\right)+\epsilon \alpha(|I|-\nu-1)\right\} \\
& \leq \max \{0,-\epsilon \alpha\} \\
& =0 .
\end{aligned}
$$

The inequality holds because $\epsilon\left(\sum_{i \in J} m_{i}-(\nu-1) d\right) \leq 0$ by (6.4) and $\alpha(|I|-\nu) \leq$ $\sum_{i \in I \backslash J}\left(d-m_{i}\right)$ by $(6.6)$.

Proposition 6.15. In the above notation, there exist non-negative numbers $\alpha_{I, \sigma_{t}} \in$ $\mathbb{Z}$ such that

$$
\left|\tilde{D}^{\prime}\right|+\alpha \tilde{\Sigma}^{\sigma}+\sum_{(I, t) \in \mathcal{C}^{\text {even }}} \alpha_{I, \sigma_{t}} E_{I, \sigma_{t}} \subseteq\left|\tilde{D}^{\sigma}\right|
$$

Proof. In order to prove the statement, we compare the strict transform, $\left|\tilde{D}^{\prime}{ }^{\sigma}\right|+\alpha \tilde{\Sigma}^{\sigma}$ with that of $D$.

Recall that, for any $1 \leq t<\nu$ and $I$ such that $|I| \geq 0$, we have $k_{I, \sigma_{t}}\left(D^{\prime}\right)=0$ by Proposition 6.14. Moreover for all $I$ and $t$, (6.4) implies that

$$
k_{I, \sigma_{t}}(\alpha \Sigma)=\alpha k_{I, \sigma_{t}}(\Sigma) \geq k_{I, \sigma_{t}}(D) .
$$

This, together with (6.5) and the computation of $k_{I}(D)$ made in the proof of Proposition 6.14, implies the following linear equivalence of divisors

$$
\tilde{D}^{\sigma} \sim \tilde{D}^{\prime}{ }^{\sigma}+\alpha \tilde{\Sigma}^{\sigma}+\sum_{(I, t) \in \mathcal{C}^{\mathrm{even}}} \alpha_{I, \sigma_{t}} E_{I, \sigma_{t}},
$$

where $\alpha_{I}:=k_{I}(\alpha \Sigma)+k_{I}\left(D^{\prime}\right)-k_{I}(D)$ if $1 \leq|I| \leq \nu$, while $\alpha_{I, \sigma_{t}}:=k_{I, \sigma_{t}}(\alpha \Sigma)-$ $k_{I, \sigma_{t}}(D)$ for $1 \leq t \leq \nu, \nu+1 \leq|I| \leq 2 \nu$. Finally, from (6.7) we obtain $a_{I, \sigma_{t}} \geq 0$, for all $I$ and $t$.

Proof of Theorem 6.10, case $n$ even. We recall that a general member of $|D|$ vanishes at every point of $J\left(L_{I}, \sigma_{t}\right)$ with multiplicity equal to $k_{I, \sigma_{t}}$ and that $\left|\tilde{D}^{\sigma}\right|$ is 
the strict transform of $D$ under the blow-up of its secant base locus. Assume that the base locus, $\operatorname{Bs}\left|\tilde{D}^{\sigma}\right|$, is non-empty. By Proposition 6.15 , we have

$$
\mathrm{Bs}\left|\tilde{D}^{\sigma}\right| \subset \operatorname{Bs}\left|\tilde{D}^{\prime}\right| \cup \tilde{\Sigma} \cup \bigcup_{(I, t) \in \mathcal{C}^{\text {even }}} E_{I, \sigma_{t}} .
$$

Note that the divisor $\left|\tilde{D}^{\prime}\right|$ is base point free by Theorem 2.1, therefore Bs $\left|\tilde{D}^{\prime}\right|$ is empty. Hence

$$
\operatorname{Bs}\left|\tilde{D}^{\sigma}\right| \subset \tilde{\Sigma} \cup \bigcup_{(I, t) \in \mathcal{C}^{\text {even }}} E_{I, \sigma_{t}} .
$$

Assume there is a base point for $\left|\tilde{D}^{\sigma}\right|$ on one of the exceptional divisors $E_{I, \sigma_{t}}$, $(I, t) \in \mathcal{C}^{\text {even }}$, or on $\tilde{\Sigma}$. This implies the existence of a point in a join $J\left(L_{I}, \sigma_{t}\right)$, or in $\Sigma$, on which the general divisor $D$ has infinitesimal tangencies. The proof of Proposition 5.3 implies that no point of $J\left(L_{I}, \sigma_{t}\right)$, nor of $\Sigma$, carries any infinitesimal information. This leads to a contradiction.

6.3. Proof of Theorem 6.10, case $n$ odd. Recall that the strict transform on $X:=X_{2 \nu+3,(0)}$ of the cone $\mathrm{J}\left(L_{\{1\}}, \sigma_{\nu}\right) \subset \mathbb{P}^{2 \nu}$ is the divisor

$$
\Gamma:=(\nu+1) H-(\nu+1) E_{1}-\nu \sum_{i=2}^{2 \nu+4} E_{i}
$$

Proposition 6.16. In the above notation, the strict transform $\tilde{\Gamma}^{\sigma}$ of $\Gamma$ on $Y^{\sigma}$ is smooth.

Proof. Recall that the singular locus of $\mathrm{J}\left(L_{\{1\}}, \sigma_{\nu}\right) \subset \mathbb{P}^{2 \nu}$ is $\mathrm{J}\left(L_{\{1\}}, \sigma_{\nu-1}\right) \subset$ $\mathrm{J}\left(L_{\{1\}}, \sigma_{\nu}\right)$ and, more precisely, the non-reduced union of all joins $\mathrm{J}\left(L_{I}, \sigma_{t}\right)$ such that $k_{I, \sigma_{t}}(\Sigma)>1$. In particular we compute the following multiplicities. For every $I$ and $t$ such that $|I|, t \geq 0$, we have

$$
k_{I, \sigma_{t}}(\Sigma)=\max \left\{0, \nu-|I|-t+1+\delta_{I}\right\},
$$

where $\delta_{I}$ is defined as

$$
\delta_{I}:=\delta_{1, I}= \begin{cases}1 & \text { if } 1 \in I, \\ 0 & \text { if } 1 \notin I .\end{cases}
$$

All subvarieties $\mathrm{J}\left(L_{I}, \sigma_{t}\right)$ such that $|I|+t \leq \nu+\delta_{I}$ have been blown-up, hence we conclude that $\pi^{\sigma}$ is a resolution of the singularities of $\Sigma$.

Choose $\beta \in \mathbb{N}$ such that

$$
\frac{k_{C}(D)}{\nu} \leq \beta \leq \min _{1 \leq i \leq 2 \nu+4}\left\{\frac{m_{1}}{\nu+\delta_{\{i\}}}, d-m_{i}\right\},
$$

where $\delta_{\{i\}}$ is the Kronecker delta defined in (6.9).

Lemma 6.17. Under the assumptions of Theorem 3.3, such an integer $\beta$ exists.

Proof. For every $i \in\{1, \ldots, 2 \nu+4\}$, we compute

$$
\epsilon k_{C}(D) \leq 2 \nu-2 \leq 2 \frac{\nu^{2}}{\nu+\delta_{\{i\}}}-\epsilon \nu \leq \epsilon \nu\left(\frac{m_{1}}{\nu+\delta_{\{i\}}}-1\right) .
$$


In the above expression, the first inequality follows from (6.4). The second inequality follows from the assumption $\epsilon \ll 1$; in fact it is enough to take $\epsilon \leq \frac{8}{n^{2}-1}=\frac{2}{\nu^{2}+\nu}$. The last inequality follows from (3.2). Furthermore we have

$$
\epsilon k_{C}(D) \leq 2 \nu-2 \leq 2 \nu-\epsilon \nu \leq \epsilon \nu\left(d-m_{i}-1\right) .
$$

The last inequality follows from (3.3).

Consider the linear system

$$
\left|D^{\prime}\right|:=|D-\beta \Gamma|=\left|d^{\prime} H-\sum_{i=1}^{2 \nu+4} m_{i}^{\prime} E_{i}\right|,
$$

with $d^{\prime}:=d-\beta(\nu+1), m_{1}^{\prime}:=m_{1}-\beta(\nu+1)$ and $m_{i}^{\prime}:=m_{i}-\beta \nu$, for all $i \in$ $\{2 \ldots, 2 \nu+4\}$. We have

$$
\left|D^{\prime}\right|+\beta \Gamma \subseteq|D|
$$

Proposition 6.18. In the above notation, the linear system $\left|D^{\prime}\right|$ is non-empty. Moreover $\left|D^{\prime}\right|$ has only linear base locus and the strict transform in $Y^{\sigma}$ of $\left|D^{\prime}\right|$ is base point free.

Proof. By (6.10), we have $0 \leq m_{i}^{\prime} \leq d$ for all $i \in\{1, \ldots, 2 \nu+4\}$. Moreover, as in the proof of Proposition 6.14, we compute

$$
k_{C}\left(D^{\prime}\right)=\max \left\{0, \epsilon\left(\sum_{i=1}^{2 \nu+4} m_{i}^{\prime}-(2 \nu+1) d^{\prime}\right)\right\}=\max \left\{0, k_{C}(D)-\beta \nu\right\}=0 .
$$

This proves the first statement.

To prove the second statement, we notice that if $|I| \leq \nu+1$ the pair $(I, 0) \in \mathcal{C}^{\text {odd }}$, while we claim that $k_{I}\left(D^{\prime}\right)=0$ if $|I| \geq \nu+2$. This and Theorem 2.1 imply the second statement. We prove the claim for $1 \in I$; the case $1 \notin I$ is similar and we leave the details to the reader. Choose $J \subset I$ with $|J|=\nu+1$ and $1 \in J$. Let us compute

$$
\begin{aligned}
\epsilon k_{I}(D) & =\max \left\{0, \epsilon\left(\sum_{i \in I} m_{i}-(|I|-1) d\right)+\epsilon \beta(|I|-\nu-2)\right\} \\
& =\max \left\{0, \epsilon\left(\sum_{i \in J} m_{i}-(\nu) d\right)+\epsilon\left(\sum_{i \in i \backslash J}\left(m_{i}-d\right)\right)+\epsilon \beta(|I|-\nu-2)\right\} \\
& \leq \max \{0,-\epsilon \beta\} \\
& =0 .
\end{aligned}
$$

The inequality holds thanks to (6.4) and (6.10).

Proposition 6.19. In the above notation, there exist non-negative numbers $\beta_{I, \sigma_{t}} \in$ $\mathbb{Z}$ such that

$$
\left|\tilde{D}^{\prime}{ }^{\sigma}\right|+\beta \tilde{\Gamma}^{\sigma}+\sum_{(I, t) \in \mathcal{C}^{\text {odd }}} \beta_{I, \sigma_{t}} E_{I, \sigma_{t}} \subseteq\left|\tilde{D}^{\sigma}\right|
$$

Proof. The proof follows the same lines as that of Proposition 6.15 and it uses Proposition 6.18. We leave the details to the reader. 
Proof of Theorem 6.10, case $n$ odd. The proof follows the same idea as that of the case $n$ even, at the end of Section 6.2.

6.3.1. The pair $(X, \epsilon D)$ is lc, for $D$ general. Notice that the canonical divisor of $Y^{\sigma}=X_{n+3,(n-2)}^{\sigma}$ is

$$
K_{Y^{\sigma}}=-(n+1) H+(n-1) \sum E_{i}+\sum_{r=1}^{n-2}(n-r-1) \sum_{\substack{I, t: \\ r_{I, \sigma_{t}}=r}} E_{I, \sigma_{t}} .
$$

We are now ready to prove Theorem 3.3 for $s=n+3$.

Proof of Theorem 3.3, case $s=n+3$. By Corollary 6.11, $\left(Y^{\sigma}, \tilde{\Delta}\right)$ is $\log$ smooth and $\pi: Y \rightarrow X$ is a $\log$ resolution of $(X, \Delta)$.

To complete the proof, similarly to the case of only linearly obstructed divisors, we are going to show that (3.2) implies

$$
\epsilon k_{I, \sigma_{t}} \leq n-|I|-2 t+1, \quad \forall I(r), 2 \leq r_{I, \sigma_{t}} \leq n-2,
$$

that in turns implies that $\operatorname{discrep}(X, \Delta) \geq-1$. This follows from the inequalities (6.4) computed in the proof of Proposition 6.8.

\section{REFERENCES}

[1] C. Araujo, C. Casagrande On the Fano variety of linear spaces contained in two odddimensional quadrics, Geom. Topol. 21 (2017), no. 5, 3009-3045.

[2] C. Araujo, A. Massarenti, Explicit log Fano structures on blow-ups of projective spaces, Proc. Lond. Math. Soc. (3) 113 (2016), no. 4, 445-473

[3] S. Bauer, Parabolic bundles, elliptic surfaces and SU(2)-representation spaces of genus zero Fuchsian groups, Math. Ann. 290 (1991), 509-526.

[4] C. Birkar, P. Cascini, C. Hacon, J. McKernan: Existence of minimal models for varieties of log general type, J. Amer. Math. Soc. 23, 405-468, (2010)

[5] M.C. Brambilla, O. Dumitrescu and E. Postinghel, On a notion of speciality of linear systems in $\mathbb{P}^{n}$, Trans. Am. Math. Soc. no. 8, 5447-5473 (2015).

[6] M. C. Brambilla, O. Dumitrescu and E. Postinghel, On the effective cone of $\mathbb{P}^{n}$ blown-up at $n+3$ points, Exp. Math. 25, no. 4, 452-465 (2016)

[7] S. Cacciola, M. Donten-Bury, O. Dumitrescu, A. Lo Giudice, J. Park, Cones of divisors of blow-ups of projective spaces, Matematiche (Catania) 66 (2011), no. 2, 153-187.

[8] A.M. Castravet, The Cox ring of $\overline{\mathcal{M}}_{0,6}$, Trans. Am. Math. Soc. no. 7, Vol. 361 (2009), 38513878.

[9] A.M. Castravet and J. Tevelev, Hilbert's 14th problem and Cox rings, Compos. Math. 142 (2006), no. 6, 1479-1498.

[10] A.M. Castravet and J. Tevelev, $\overline{\mathcal{M}}_{0, n}$ is not a Mori Dream Space, Duke Math. J., 164, no. 8 (2015), 1641-1667

[11] C. Ciliberto, Geometrical aspects of polynomial interpolation in more variables and of Waring's problem, European Congress of Mathematics, Vol. I (Barcelona, 2000), 289-316, Progr. Math., 201, Birkhäuser, Basel, 2001.

[12] O. Dumitrescu and E. Postinghel, Vanishing theorems for linearly obstructed divisors, J. Algebra, 477, 312-359 (2017)

[13] O. Dumitrescu and E. Postinghel, Positivity of divisors on blown-up projective spaces, I, preprint arXiv:1506.04726

[14] S. Fukuda, On numerically effective log canonical divisors, Int. J. Math. Math. Sci. 30, no. $9,521-531,(2002)$

[15] R. Fröberg, An inequality for Hilbert series of graded algebras, Math. Scand. 56 , no. 2, $117-144,(1985)$ 
[16] A. Gibney, S. Keel and I. Morrison, Towards the ample cone of $\bar{M}_{g, n}$, J. Amer. Math. Soc. $15,273-294,(2002)$

[17] Y. Gongyo, Remarks on the non-vanishing conjecture, Algebraic geometry in east Asia-Taipei 2011, 107-116, Adv. Stud. Pure Math., 65, Math. Soc. Japan, Tokyo (2015).

[18] J. Gonzalez, K. Karu, Some non-finitely generated Cox rings, Compos. Math. 152 (2016), no. 5, 984-996.

[19] C. D. Hacon, J. McKernan,C. Xu, ACC for log canonical thresholds, Ann. of Math. (2) 180 (2014), no. 2, 523--571

[20] Y. Hu and S. Keel, Mori Dream Spaces and GIT, Michigan Math. J. 48, 331-348 (2000)

[21] A. Iarrobino, Inverse system of symbolic power III. Thin algebras and fat points, Compositio Math. 108 (1997), no. 3, 319-356.

[22] M. Kapranov, Veronese curves and Grothendieck-Knudsen moduli space $\overline{M_{0, n}}$, J. Algebraic Geom. 2 (1993), no. 2, pp. 239-262.

[23] S. Keel, K. Matsuki and J. McKernan, Log abundance theorem for threefolds, Duke Math. J. 75 , no. $1,99-119,(1994)$

[24] S. Keel and J. McKernan, Contraction of extremal rays on $\overline{M_{0, n}}$, arXiv:alg-geom/9607009, (1996).

[25] J. Kollár and S. Mori, Birational geometry of algebraic varieties, Cambridge Tracts in Mathematics, vol. 134, Cambridge University Press, Cambridge, (1998), With the collaboration of C. H. Clemens and A. Corti, Translated from the 1998 Japanese original.

[26] J. Hausen, S. Keicher, A. Laface On blowing-up the weighted projective plane, Math. Z. 290 (2018), no. 3-4, 1339-1358.

[27] A. Losev, Y. Manin, New moduli spaces of pointed curves and pencils of flat connections, Michigan Math. J. Volume 48, Issue 1, 2000, 443-472.

[28] H.B. Moon, S.B. Yoo, Birational Geometry of the Moduli Space of Rank 2 Parabolic Vector Bundles on a Rational Curve, Int. Math. Res. Not. IMRN 2016, no. 3, 827-859.

[29] S. Mukai, Counterexample to Hilberts fourteenth problem for the 3-dimensional additive group, RIMS preprint \#1343, Kyoto, (2001)

[30] S. Mukai, Finite generation of the Nagata invariant rings in A-D-E cases, 2005, RIMS Preprint \# 1502.

[31] G. Veronese, Behandlung der projectivischen Verhältnisse der Räume von verschiedenen Dimensionen durch das Princip des Prjjicirens und Schneidens, (German), Math. Ann. 19 (1881), no. 2, 161-234.

Olivia Dumitrescu: Central Michigan University, Pearce Hall 209, Mt. Pleasant, MiCHIGAN 48859, US,

and Simion Stollow Institute of Mathematics, Romanian Academy, 21 Calea Grivitei Street, 010702 Bucharest, Romania

Email address: dumit1om@cmich.edu

Elisa Postinghel: Department of Mathematical Sciences, Loughborough University, LE11 3TU, UK

Email address: E.Postinghel@lboro.ac.uk 